\title{
Review \\ Current Knowledge on the Fungal Degradation Abilities Profiled through Biodeteriorative Plate Essays
}

\author{
João Trovão ${ }^{1, *}$ and António Portugal ${ }^{1,2}$ (D) \\ 1 Centre for Functional Ecology, Department of Life Sciences, University of Coimbra, \\ 3004-531 Coimbra, Portugal; aportuga@bot.uc.pt \\ 2 Fitolab-Laboratory for Phytopathology, Instituto Pedro Nunes, 3030-199 Coimbra, Portugal \\ * Correspondence: jtrovaosb@gmail.com
}

Citation: Trovão, J.; Portugal, A. Current Knowledge on the Fungal Degradation Abilities Profiled through Biodeteriorative Plate Essays. Appl. Sci. 2021, 11, 4196. https:// doi.org/10.3390/app11094196

Academic Editor: Daniela Isola

Received: 26 March 2021

Accepted: 27 April 2021

Published: 5 May 2021

Publisher's Note: MDPI stays neutral with regard to jurisdictional claims in published maps and institutional affiliations.

Copyright: (c) 2021 by the authors. Licensee MDPI, Basel, Switzerland. This article is an open access article distributed under the terms and conditions of the Creative Commons Attribution (CC BY) license (https:// creativecommons.org/licenses/by/ $4.0 /)$.

\begin{abstract}
Fungi are known to contribute to the development of drastic biodeterioration of historical and valuable cultural heritage materials. Understandably, studies in this area are increasingly reliant on modern molecular biology techniques due to the enormous benefits they offer. However, classical culture dependent methodologies still offer the advantage of allowing fungal species biodeteriorative profiles to be studied in great detail. Both the essays available and the results concerning distinct fungal species biodeteriorative profiles obtained by amended plate essays, remain scattered and in need of a deep summarization. As such, the present work attempts to provide an overview of available options for this profiling, while also providing a summary of currently known fungal species putative biodeteriorative abilities solely obtained by the application of these methodologies. Consequently, this work also provides a series of checklists that can be helpful to microbiologists, restorers and conservation workers when attempting to safeguard cultural heritage materials worldwide from biodeterioration.
\end{abstract}

Keywords: biodeterioration; cultural heritage; deteriorative action; enzymatic activity; fungi

\section{Introduction}

The Fungal Kingdom comprises a highly diverse eukaryotic group able to inhabit every ecological niche available on the Planet [1]. The growth and biological activity of fungal species in cultural heritage materials is known to develop serious damages by means of biodeterioration (the undesirable modifications of a valuable material occurring by the action of living organisms) [2,3]. Fungi are highly versatile, ubiquitous, chemoheterotrophic microorganisms, being able to grow in a vast number of materials and contributing to the development of various biodeterioration phenomena [2,3]. Such modifications are a result from fungal species settling, development and exploitation of various organic and inorganic compounds present in historic art-pieces and monuments [2-15]. The fungal biodeterioration of books, paper, parchment, textiles, photographs, paintings, sculptures and wooden materials occurs due to the aesthetic modifications, mechanical pressure and exoenzymatic action [2]. Various components of these materials such as cellulose, collagen, linen, glues, inks, waxes and organic binders can be oxidized, hydrolyzed, dissolved, stained or structurally modified as a result of the action of fungal enzymes, pigments and organic acids [2,3,7-11]. A typical and widely known example of these phenomena is known as "foxing", the development of red-brownish localized spots, hypothesized to be a result from fungal proliferation and metabolization of organic acids, oligosaccharides and proteic compounds that can stain and modify the constituent materials of many paper-based and photographic supports [3,8,13]. Another example of microorganism's attack of organic materials is related to the biodeterioration of human remains, mummies and funerary materials, where opportunistic, saprotrophic and highly cellulolytic and proteolytic taxa are able to thrive and trough their actions severely alter them $[2,14,15]$. Complementarily, historic relics mainly composed of inorganic components such as stone, 
frescoes, glass and ceramics can also suffer deep aesthetical, physical and chemical modifications resulting from fungal grow and action $[2-6,12,16]$. In these supports, deterioration is caused by hyphae penetration into the substrate, the production and release of extracellular destructive organic acids, enzymes and metabolites and by the the formation of distinct colored outlines as a result of fungi high pigment contents, contribution to biofilm development and chemical reactions with inorganic compounds [2-6].

Due to the known biodeterioration problems arising from their proliferation, the accurate species identification and a consequent deteriorative profiling of isolates are crucial steps towards the development and the establishment of proper protective measures for the diverse cultural heritage treasures around the world. With the recent development of innovative culture independent methodologies such as -omics technologies, molecular data is becoming increasingly more valuable for the identification of the microbes, the characterization of their metabolic functions and their deteriorative byproducts [17]. Methodologies such as metagenomics, transcriptomics, metabolomics and proteomics revolutionized the field and are increasingly allowing understanding of microbial diversity, but also species specific and holistic contributions to various materials biodeterioration phenomena [17]. These methods are particularly relevant considering that traditional cultivation dependent methodologies hold the disadvantage of being unable to correctly infer microorganism's abundance and only allow the study of active forms, failing to provide information regarding viable non-culturable and non-viable forms [17-27]. Nonetheless, classical culture dependent methodologies still offer an important advantage when compared with modern methodologies, especially when considering that the isolation of microbes allows their natural biodeteriorative profiles to be studied in great detail. Culture media plates modified to specify a positive biodeteriorative ability upon the microorganism development and deteriorative action (see Figure 1 for examples) can provide valuable data that allow the evaluation of the microorganism's putative risks to cultural heritage materials. Moreover, they also offer a highly informative, rapid and low-cost platform [28] that can help in a quick and focused decision-making process aiming to protect valuable artifacts. Currently, plate assays aiming to identify fungal deteriorative characteristics, such as calcium carbonate solubilization, mineralization and various enzymatic activities, have been proposed and somewhat widely used.

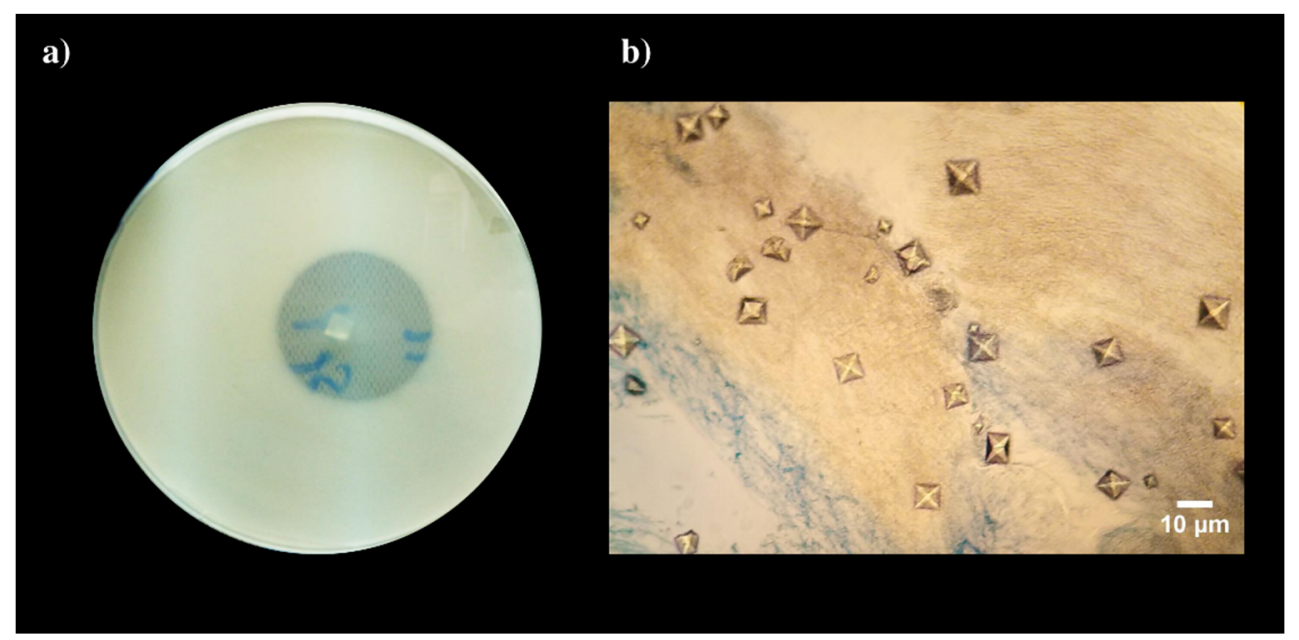

Figure 1. Examples of fungal species biodeteriogenic abilities detected through plate assays: (a) Calcium carbonate dissolution visualized by the development of a halo around colonies in $\mathrm{CaCO}_{3}$ glucose agar; and (b) calcium oxalates crystals developing around fungal mycelium growing in Malt extract agar containing $\mathrm{CaCO}_{3}$.

Although differences among distinct isolates, assays and incubation conditions are known and expected, the available literature concerning distinctive fungal species deteriorative profiles obtained using such methodologies remains pending a deep summa- 
rization. With this in mind, this work aims to provide an overview of available plate assays, as well as the fungal putative biodeteriorative profiles obtained solely through such tests so far. In addition, we also aimed at providing a series of quick and straight forward checklists that can be consulted by microbiologists, restorers and conservation staff, when working to safeguard important cultural heritage materials worldwide. These checklists were also annotated to contain currently accepted fungal names according to Mycobank (www.mycobank.org, last accessed on 26 April 2021) and Index Fungorum (www.indexfungorum.org, last accessed on 26 April 2021) in order to ensure an updated identification for fungi displaying such profiles, and to facilitate information sharing in the future.

\section{Calcium Carbonate Solubilization or Dissolution}

One of the greatest fungal effects on stone monuments is credited to their secretion of inorganic and organic acids that can alter the material properties [2-4,29-32]. In fact, carbonate weathering has been consistently linked to the excretion and action of these metabolites [33-35]. Evaluation of fungal calcium carbonate solubilization abilities in cultural heritage scenarios has been helpful to study the biodeteriorative contribution of isolates retrieved from air, mural paintings, wooden art objects, frescoes, catacombs, bricks, concrete, buildings and various limestone and plaster monuments and museums [28,33,35-44]. Fungal calcium carbonate solubilization ability screening is usually conducted with $\mathrm{CaCO}_{3}$ glucose agar and adapted formulations [33]. Nonetheless, the application of Malt extract agar and Reasoner's 2A agar amended with $\mathrm{CaCO}_{3}$ (CMEA and CR2A) has also been successfully achieved [35]. Moreover, Kiyuna and colleagues [37] also highlighted the utility of Glucose Yeast extract calcium carbonate agar (GYC) [45] for such evaluation. Positive $\mathrm{CaCO}_{3}$ dissolution is usually evaluated by the visualization of a halo around the growing colony after a period of incubation. In addition, calcium carbonate solubilization screening can also be conducted coupled with the evaluation of the media $\mathrm{pH}$ modifications. For this purpose, Creatine Sucrose agar (CREA) [46] followed by the analysis of medium color changes around growing colonies, or liquid media according to the formulations provided by Borrego and colleagues [47] followed by $\mathrm{pH}$ analysis, can also be applied. A quick overview of the known fungal species able of $\mathrm{CaCO}_{3}$ dissolution points that isolates from more than fifty species have been found to display this biodeteriorative profile, with the great majority of them being Aspergillus and Penicillium species (Table 1). Both genera are known important biodeteriogens, producing various acidic molecules and contributing to the deterioration of materials [28,48]. The detection of species from these genera (as well as others for example, from Pestalotiopsis and Talaromyces among others) might indicate a putative threat to acid susceptible resources, such is the case of stone structures, mural paintings and frescoes.

Table 1. Overview of fungal species for which isolates have been identified as having $\mathrm{CaCO}_{3}$ dissolution abilities in biodeteriorative plate essays.

\begin{tabular}{|c|c|c|}
\hline Current Species Name & Original Study Focus & References \\
\hline Acremonium charticola (Lindau) W. Gams & Limestone and plaster monuments and museums & [39] \\
\hline Actinomucor elegans (Eidam) C.R. Benjamin and Hesseltine & Mural paintings & [41] \\
\hline Alternaria alternata (Fr.) Keissl. & Mural paintings & [41] \\
\hline $\begin{array}{l}\text { Annulohypoxylon stygium (Lév.) Y.M. Ju, J.D. Rogers and } \\
\text { H.M. Hsie }\end{array}$ & Mayan buildings & [35] \\
\hline Aspergillus amstelodami (L. Mangin) Thom and Church & Wooden art objects & [36] \\
\hline Aspergillus awamori Nakaz. & Wooden art objects & [36] \\
\hline $\begin{array}{c}\text { Aspergillus europaeus Hubka, A. Nováková, Samson, } \\
\text { Houbraken, Frisvad and M. Kolařík }\end{array}$ & Frescoes and air & [38] \\
\hline Aspergillus glaucus (L.) Link & Limestone tomb & [43] \\
\hline Aspergillus nidulans (Eidam) G. Winter & Mural paintings & [41] \\
\hline Aspergillus niger Tiegh & Wooden art objects, frescoes, air, brick and concrete & {$[28,36,38,40]$} \\
\hline Aspergillus versicolor (Vuill.) Tirab & $\begin{array}{l}\text { Wooden art objects, mural paintings, limestone, } \\
\text { plaster monuments and museums }\end{array}$ & {$[36,39,41]$} \\
\hline
\end{tabular}


Table 1. Cont.

\begin{tabular}{|c|c|c|}
\hline Current Species Name & Original Study Focus & References \\
\hline Aspergillus westerdijkiae Frisvad and Samson & Limestone tomb & [43] \\
\hline Botrytis cinerea Pers. & Limestone tomb & [43] \\
\hline Cephalotrichum Link $^{1}$ & Catacombs & [33] \\
\hline Cladosporium Link & Etruscan hypogeal tombs & [44] \\
\hline Cladosporium sphaerospermum Penz. & Mural paintings & [41] \\
\hline Cyphellophora G.A. de Vries & Etruscan hypogeal tombs & [44] \\
\hline Cyphellophora olivacea (W. Gams) Réblová \& Unter. & Etruscan hypogeal tombs & [44] \\
\hline Exophiala J.W. Carmich. & Etruscan hypogeal tombs & [44] \\
\hline Kendrickiella phycomyces (Auersw.) K. Jacobs and M.J. Wingf. & Mural paintings in Tumuli & [37] \\
\hline Lasiodiplodia theobromae (Pat.) Griffon and Maubl. & Mayan buildings & [35] \\
\hline Lecanicillium W. Gams and Zare & Limestone and plaster monuments and museums & [39] \\
\hline Paecilomyces Bainier & Mayan buildings and catacombs & {$[33,35]$} \\
\hline $\begin{array}{l}\text { Parengyodontium album (Limber) C.C. Tsang, J.F.W. Chan, } \\
\text { W.M. Pong, J.H.K. Chen, A.H.Y. Ngan, M. Cheung, C.K.C. } \\
\text { Lai, D.N.C. Tsang, S.K.P. Lau and P.C.Y. Woo }\end{array}$ & Dolomitic limestone wall & [42] \\
\hline Penicillium angulare S.W. Peterson, E.M. Bayer and Wicklow & Dolomitic limestone wall & [42] \\
\hline Penicillium aurantiogriseum Dierckx & Mural paintings & [41] \\
\hline Penicillium bilaiae Chalab. & Frescoes and air & [38] \\
\hline Penicillium brevicompactum Dierckx & Air, limestone tomb and dolomitic limestone wall & {$[28,42,43]$} \\
\hline Penicillium chrysogenum Thom & $\begin{array}{l}\text { Wooden art objects, air, mural paintings, limestone } \\
\text { tomb, dolomitic limestone wall, limestone and } \\
\text { plaster monuments and museums }\end{array}$ & $\begin{array}{c}{[28,36,39,41-} \\
43]\end{array}$ \\
\hline Penicillium commune Thom & Frescoes, air and mural paintings & {$[38,40,41]$} \\
\hline Penicillium crustosum Thom & Dolomitic limestone wall & [42] \\
\hline Penicillium glabrum (Wehmer) Westling & $\begin{array}{l}\text { Wooden art objects, air, dolomitic limestone wall } \\
\text { and limestone tomb }\end{array}$ & {$[28,36,42]$} \\
\hline Penicillium griseofulvum Dierckx & Frescoes and air & [38] \\
\hline Penicillium lanosum Westling & Frescoes and air & [38] \\
\hline Penicillium Link & $\begin{array}{l}\text { Wooden art objects, air, frescoes, concrete and } \\
\text { bricks }\end{array}$ & {$[36,40]$} \\
\hline Penicillium oxalicum Currie and Thom & Mayan buildings & [35] \\
\hline Penicillium polonicum K.W. Zaleski & Mural paintings & [41] \\
\hline Penicillium rubens Biourge & Frescoes and air & [38] \\
\hline Penicillium scabrosum Frisvad, Samson and Stolk & Dolomitic limestone wall & [42] \\
\hline Penicillium solitum Westling & Air & [28] \\
\hline Penicillium viridicatum Westling & Air & [28] \\
\hline Periconia byssoides Pers. & Dolomitic limestone wall & [42] \\
\hline Pestalotiopsis maculans (Corda) Nag Raj & Mayan buildings & [35] \\
\hline Pestalotiopsis microspora (Speg.) G.C. Zhao and Nan Li & Mayan buildings & [35] \\
\hline Pseudogymnoascus pannorum (Link) Minnis and D.L. Lindner & Limestone and plaster monuments and museums & [39] \\
\hline Rosellinia De Not. & Mayan buildings & [35] \\
\hline Sclerotinia Fuckel & Air and frescoes & [36] \\
\hline Sclerotinia sclerotiorum (Lib.) de Bary & Air and frescoes & [36] \\
\hline $\begin{array}{c}\text { Talaromyces amestolkiae N. Yilmaz, Houbraken, Frisvad and } \\
\text { Samson }\end{array}$ & Dolomitic limestone wall & [42] \\
\hline $\begin{array}{c}\text { Talaromyces sayulitensis Visagie, N. Yilmaz, Seifert and } \\
\text { Samson }\end{array}$ & Air & [28] \\
\hline Trichocladium canadense S. Hughe & Mayan buildings & [35] \\
\hline Trichoderma Pers. & Mayan buildings & [35] \\
\hline Valsaria spartii Maubl. & Dolomitic limestone wall & [42] \\
\hline Xylaria Hill ex Schrank ${ }^{2}$ & Mayan buildings & [35] \\
\hline
\end{tabular}




\section{Mineralization or Crystallization Development}

Calcium carbonate solubilization by the action of fungal acids can often occur coupled with the recrystallization of minerals in the substrate [2,3,5,6,49-51]. Such mineralization singularities can lead to the development of various biodeterioration phenomena $[52,53]$. They occur from the reactions of secreted acids (especially oxalic acid) with stone cations [32] and often result in the formation of carbonates and/or calcium magnesium oxalates $[5,6]$. Characterization of fungal crystallization abilities in cultural heritage scenarios has been helpful to study the biodeteriorative contribution of isolates retrieved from air, limestone monuments, stone stela, wall and mural paintings [35,41-43,53-56]. Fungal mineralization ability screening is usually conducted using B4 (with calcium acetate) or modified B4 (with calcium carbonate) media and adapted formulations [57]. Moreover, $\mathrm{CaCO}_{3}$ modified Malt agar, Nutrient agar (NA) with $\mathrm{CaCl}_{2}$ and the above mentioned CMEA and CR2A media have also been found useful for such purposes [35,42,43,58,59]. Positive mineralization development is usually evaluated by the microscopical visualization of neo-formed minerals around or in fungal hyphae after a period of incubation. Moreover, further characterization of these crystals can also be achieved by applying analytical methodologies such as X-ray powder diffraction (XRD) and/or energy dispersive X-ray spectroscopy (EDS) in conjunction with scanning electron microscopy (SEM) methodologies. So far, circa sixty species have been found to display mineralization abilities in plate essays and, as similarly found for fungal calcium carbonate dissolution, multiple Aspergillus and Penicillium species have also denoted this biodeteriorative profile (Table 2). Such findings can be correlated with their long-known abilities to secrete oxalic acid, among various other acids [41]. Nonetheless, a relevant number of species from genera Alternaria, Cladosporium, Colletotrichum, Pestalotiopsis and Trichoderma putatively displaying these biodeteriorative abilities can also be verified. Typical minerals detected include calcium carbonate in the form of calcite and vaterite-calcite, weddellite, whewellite, hydroxyapatite, hydrocerussite, pyromorphite, phosphate and other still unidentified calcium oxalates and minerals. The detection of species from these genera might indicate a putative threat to materials highly susceptible to fungal acidolysis and biomineralization, such is the case of limestone monuments and murals $[5,6,60]$.

Table 2. Overview of fungal species for which isolates have been identified as displaying mineralization abilities in biodeteriorative plate essays.

\begin{tabular}{|c|c|c|c|}
\hline Current Species Name & Mineral Details & Original Study Focus & References \\
\hline $\begin{array}{c}\text { Actinomucor elegans (Eidam) C.R. Benjamin } \\
\text { and Hesseltine }\end{array}$ & Cc, Wd & Mural paintings & [41] \\
\hline Aeminium ludgeri J. Trovão, I. Tiago and A. Portugal & $\mathrm{Cc}$ & Dolomitic limestone wall & [42] \\
\hline Alternaria alternata (Fr.) Keissl. & Unk & Air and wall paintings & [56] \\
\hline Alternaria infectoria E.G. Simmons & Unk & Air and wall paintings & [56] \\
\hline $\begin{array}{c}\text { Annulohypoxylon stygium (Lév.) Y.M. Ju, J.D. Rogers } \\
\text { and H.M. Hsie }\end{array}$ & $\mathrm{Cc}, \mathrm{Wd}$ & Mayan buildings & [35] \\
\hline Ascochyta medicaginicola Qian Chen and L. Cai ${ }^{1}$ & Unk & Air and wall paintings & [56] \\
\hline Aspergillus aureolatus Munt.-Cvetk. and Bata & $\mathrm{Cc}$ & Air and wall paintings & [56] \\
\hline $\begin{array}{c}\text { Aspergillus europaeus Hubka, A. Nováková, Samson, } \\
\text { Houbraken, Frisvad and M. Kolařík }\end{array}$ & Unk & Air and wall paintings & {$[56]$} \\
\hline $\begin{array}{c}\text { Aspergillus flavipes (Bainier and R. Sartory) Thom } \\
\text { and Church }\end{array}$ & Unk & Air and wall paintings & [56] \\
\hline Aspergillus flavus Link & $\mathrm{Cc}, \mathrm{Wd}$ & Air and wall paintings & [56] \\
\hline Aspergillus glaucus (L.) Link & Unk CO & Limestone tomb & [54] \\
\hline Aspergillus niger Tiegh & Unk, Wh & $\begin{array}{l}\text { Limestone monument, air } \\
\text { and wall paintings }\end{array}$ & {$[53,56]$} \\
\hline Aspergillus ostianus Wehmer & Cc, Wd & Air and wall paintings & [56] \\
\hline $\begin{array}{c}\text { Aspergillus pallidofulous Visagie, Varga, Frisvad } \\
\text { and Samson }\end{array}$ & $\mathrm{Cc}$ & Air and wall paintings & [56] \\
\hline Aspergillus parasiticus Speare & $\mathrm{Cc}, \mathrm{Wd}$ & Air and wall paintings & [56] \\
\hline
\end{tabular}


Table 2. Cont.

\begin{tabular}{|c|c|c|c|}
\hline Current Species Name & Mineral Details & Original Study Focus & References \\
\hline Aspergillus westerdijkiae Frisvad and Samson & Unk CO & Limestone tomb & [54] \\
\hline $\begin{array}{c}\text { Bionectria ochroleuca (Schwein.) Schroers } \\
\text { and Samuels }\end{array}$ & Cc, Wh & Stone stela & {$[54]$} \\
\hline $\begin{array}{c}\text { Botryotrichum murorum (Corda) X. Wei Wang } \\
\text { and Samson }{ }^{2}\end{array}$ & Cc, Unk & $\begin{array}{l}\text { Air, wall paintings and } \\
\text { stone stela }\end{array}$ & {$[54,56]$} \\
\hline Botrytis cinerea Pers. & Unk CO & Limestone tomb & [54] \\
\hline Chaetomium ancistrocladum Udagawa and Cain & Unk & Air and wall paintings & [56] \\
\hline Cladosporium cladosporioides (Fresen.) G.A. de Vries & Unk & Air and wall paintings & [56] \\
\hline Cladosporium oxysporum Berk. and M.A. Curtis & Cc, Wd & Air and wall paintings & [56] \\
\hline Cladosporium sphaerospermum Penz. & Cc, Wd & Mural paintings & [41] \\
\hline Cladosporium uredinicola Speg. & $\mathrm{Cc}$ & Air and wall paintings & [56] \\
\hline Colletotrichum acutatum J.H. Simmonds & Cc (Vaterite), Cc, Hap, Phosp & Limestone monument & {$[53,55]$} \\
\hline Colletotrichum gloeosporioides (Penz.) Penz. and Sacc. & Cc (Vaterite) & Limestone monument & {$[53]$} \\
\hline Epicoccum nigrum Link & $\mathrm{Cc}, \mathrm{Wd}$ & Air and wall paintings & [56] \\
\hline Fusarium fujikuroi Nirenberg ${ }^{3}$ & $\mathrm{Cc}$ & Air and wall paintings & [56] \\
\hline $\begin{array}{c}\text { Fusarium proliferatum (Matsush.) Nirenberg ex } \\
\text { Gerlach and Nirenberg }\end{array}$ & $\mathrm{Cc}$ & Stone stela & [54] \\
\hline Lasiodiplodia theobromae (Pat.) Griffon and Maubl. & Unk & Mayan buildings & [35] \\
\hline Leptosphaeria avenaria G.F. Weber ${ }^{4}$ & $\mathrm{Cc}$ & Air and wall paintings & [56] \\
\hline Mucor fragilis Bainier & Cc, Wd & Dolomitic limestone wall & [42] \\
\hline Paecilomyces Bainier & Unk & Mayan buildings & [35] \\
\hline $\begin{array}{c}\text { Penicillium angulare S.W. Peterson, E.M. Bayer } \\
\text { and Wicklow }\end{array}$ & Cc, Wd, Wh & Dolomitic limestone wall & [42] \\
\hline Penicillium atrosanguineum B.X. Dong ${ }^{5}$ & Cc, Wd & Air and wall paintings & [56] \\
\hline Penicillium bilaiae Chalab. & Cc, Wd & Air and wall paintings & {$[56]$} \\
\hline Penicillium brevicompactum Dierckx & Cc, Wd & Dolomitic limestone wall & [42] \\
\hline Penicillium chrysogenum Thom & Cc, Wd, Unk CO & $\begin{array}{l}\text { Limestone tomb and } \\
\text { mural paintings }\end{array}$ & {$[41,43]$} \\
\hline Penicillium commune Thom & Cc, Wd & $\begin{array}{l}\text { Air, wall and mural } \\
\text { paintings }\end{array}$ & {$[41,56]$} \\
\hline Penicillium crustosum Thom & Cc, Wd & Stone stela & [54] \\
\hline Penicillium glabrum (Wehmer) Westling & Cc, Wd, Wh & Dolomitic limestone wall & [42] \\
\hline Penicillium griseofulvum Dierckx & Unk & Air and wall paintings & [56] \\
\hline Penicillium lanosum Westling & Cc, Wd, Unk & Air and wall paintings & [56] \\
\hline Penicillium oxalicum Currie and Thom & Cc, Wd, Wh & $\begin{array}{l}\text { Mayan buildings and } \\
\text { limestone monument }\end{array}$ & {$[35,53]$} \\
\hline Penicillium polonicum K.W. Zaleski & Cc, Wd & Mural paintings & [41] \\
\hline Penicillium rubens Biourge & Unk & Air and wall paintings & [56] \\
\hline Periconia byssoides Pers. & $\mathrm{Cc}$ & Dolomitic limestone wall & [42] \\
\hline Pestalotiopsis maculans (Corda) Nag Raj & Unk & Mayan buildings & [35] \\
\hline $\begin{array}{c}\text { Pestalotiopsis microspora (Speg.) G.C. Zhao and } \\
\text { Nan Li }\end{array}$ & Cc, Wd, Wh & Mayan buildings & [35] \\
\hline $\begin{array}{l}\text { Phialemonium inflatum (Burnside) Dania García, } \\
\text { Perdomo, Gené, Cano and Guarro } 6\end{array}$ & Cc, Cc (Vaterite), Hyd, Pyr & Stalactite in building & [59] \\
\hline Plectosphaerella cucumerina (Lindf.) W. Gams & Cc, Hyd, Pyr & Stalactite in building & [59] \\
\hline Rhizoctonia solani J.G. Kühn ${ }^{7}$ & Unk & Air and wall paintings & [56] \\
\hline Rosellinia De Not. & Cc, Wd & Mayan buildings & [35] \\
\hline Sclerotinia sclerotiorum (Lib.) de Bary & Cc, Wd & Air and wall paintings & [56] \\
\hline Stereum hirsutum (Willd.) Pers. & Cc, Wd & Dolomitic limestone wall & {$[42]$} \\
\hline Trichocladium canadense S. Hughes & Unk & Mayan buildings & [35] \\
\hline Trichoderma atroviride P. Karst. & $\mathrm{Cc}, \mathrm{Wd}$ & Dolomitic limestone wall & [42] \\
\hline Trichoderma harzianum Rifai & $\mathrm{Cc}$ & Stone stela & [54] \\
\hline Trichoderma Pers. & Unk & Mayan buildings & [35] \\
\hline Xylaria Hill ex Schrank ${ }^{8}$ & Unk & Mayan buildings & {$[35]$} \\
\hline
\end{tabular}

Previously identified as: ${ }^{1}$ Phoma medicaginis; ${ }^{2}$ Chaetomium murorum; ${ }^{3}$ Gibberella moniliformis; ${ }^{4}$ Phaeosphaeria avenaria; ${ }^{5}$ Penicillium manginii; ${ }^{6}$ Paecilomyces inflatus; ${ }^{7}$ Thanatephorus cucumeris; ${ }^{8}$ Hypoxylon sp.; Cc—calcium carbonate; Wd-weddellite; Wh-whewellite; Hap-hydroxyapatite; Hyd—hydrocerussite; Phosp—phosphate; Pyr—pyromorphite; Unk CO—Unidentified calcium oxalates; Unk— Unidentified mineralization's. 


\section{Enzymatic Action}

Fungal ligninolytic action is often considered a threat to wooden structures [61-66]. Cultural heritage materials constructed with these materials can be affected by fungal hyphae penetration but also by the action of various exoenzymes [67]. Moreover, brown and white rot fungi are known to contribute to these substrates' deterioration and degradation in various contexts [68]. So far, fungal ligninolytic ability characterization in cultural heritage scenarios has been helpful to study the biodeteriorative contribution of isolates retrieved from air, wooden materials and art objects $[61,69,70]$. Ligninolytic ability screening can be conducted using media with Azure B (lignin peroxidase), Phenol Red (Mn peroxidase), Remazol Brilliant Blue R (laccase) [71-74] or, alternatively, by applying Potato Dextrose agar supplemented with guaiacol (PDA-guaiacol) [61,70]. Positive ligninolytic ability is usually evaluated by the clearance of the media specific color (Azure B, Phenol Red and Remazol Brilliant Blue R) or by the development of reddish-brown zones (PDA-guaiacol) after a period of incubation. As pointed by Pangallo and colleagues [36,69], data regarding ligninolytic abilities of filamentous fungi in biodeterioration contexts is still somewhat scarce. Nonetheless, as evidenced by Table 3, almost thirty species have been found to display these biodeteriorative abilities. Moreover, mainly species of genera Aspergillus, Chaetomium, Cladosporium and Penicillium represent the bulk of the currently studied lignin deteriorating fungi. As such, the detection of species from these genera might indicate a putative threat to lignin materials, such as the case of some types of paper and wood art pieces and objects.

Table 3. Overview of fungal species for which isolates have been identified as displaying ligninolytic abilities in biodeteriorative plate essays.

\begin{tabular}{|c|c|c|}
\hline Current Species Name & Original Study Focus & References \\
\hline Alternaria consortialis (Thüm.) J.W. Groves and S. Hughes ${ }^{1}$ & Wooden art objects & [36] \\
\hline Arthrinium phaeospermum (Corda) M.B. Ellis & Wooden art objects & {$[36]$} \\
\hline Aspergillus amstelodami (L. Mangin) Thom and Church ${ }^{2}$ & Wooden art objects & {$[36]$} \\
\hline Aspergillus awamori Nakaz. & Wooden art objects & [36] \\
\hline Aspergillus fischeri Wehmer ${ }^{3}$ & Wooden art objects & [36] \\
\hline Aspergillus flavus Link & Wooden art objects & {$[36]$} \\
\hline Aspergillus fumigatus Fresen. & Wooden art objects & {$[36]$} \\
\hline Aspergillus niger Tiegh & Wooden art objects & [36] \\
\hline Aspergillus terreus Thom & Wooden art objects and air & {$[36,69]$} \\
\hline Aspergillus ustus (Bainier) Thom and Church & Wooden art objects & {$[36]$} \\
\hline Aspergillus versicolor (Vuill.) Tirab. & Wooden art objects & {$[36]$} \\
\hline Beauveria bassiana (Bals.-Criv.) Vuill. & Wooden art objects & {$[36]$} \\
\hline Chaetomium elatum Kunze & Wooden art objects & [36] \\
\hline Chaetomium globosum Kunze & Wooden art objects and air & {$[36,69]$} \\
\hline Cladosporium cladosporioides (Fresen.) G.A. de Vries & Wooden objects and air & [69] \\
\hline Cladosporium herbarum (Pers.) Link & Wooden art objects & {$[36]$} \\
\hline Cladosporium Link & Wooden art objects & {$[36]$} \\
\hline Hypochnicium J. Erikss. & Wooden materials & {$[61]$} \\
\hline "Neocosmospora" solani (Mart.) L. Lombard and Crous 4 & Wooden materials & {$[70]$} \\
\hline Penicillium chrysogenum Thom & Wooden art objects & [36] \\
\hline Penicillium expansum Link & Wooden art objects & {$[36]$} \\
\hline Penicillium glabrum (Wehmer) Westling & Wooden art objects & [36] \\
\hline Penicillium herquei Bainier and Sartory & Wooden art objects & [36] \\
\hline Penicillium Link & Wooden art objects & [36] \\
\hline Penicillium sacculum E. Dale & Wooden art objects & {$[36]$} \\
\hline Pseudogymnoascus pannorum (Link) Minnis and D.L. Lindner ${ }^{5}$ & Wooden art objects & [36] \\
\hline Trichoderma viride Pers. & Wooden art objects & [36] \\
\hline
\end{tabular}

Previously identified as: ${ }^{1}$ Alternaria consortiale; ${ }^{2}$ Eurotium amstelodami ${ }^{3}$ Neosartorya fischeri; ${ }^{4}$ Fusarium solani; ${ }^{5}$ Chrysosporium pannorum.

Fungi can also have an important role in the attack of animal-based objects, adhesives and additives. Textile materials such as silk and wool can suffer microbial mediated 
biodeterioration processes by the action of deteriorating enzymes [75,76]. In particular, the silks fibroin and sericin can both be the target of microbial attack [77]. Moreover, wool keratins can also be the target of attack by microbes [77]. Evaluation of fibroinolytic and keratinolytic action in cultural heritage scenarios has been helpful to study mummies, funeral clothes and accessories biodeterioration [78-80]. Moreover, fungal chitinolytic and pectinolytic action has also been pinpointed as a threat to Ancient Yemeni mummies preserved with diverse organic compounds [81]. Additionally, esterease action profiling has also been helpful to study isolates retrieved from wax seals, air, textiles and human remains [82-84]. Fibroinolytic screening can be conducted using fibroin agar, with the fibroinolytic action being evaluated by the isolates ability to grow in the culture-amended plates [85]. Moreover, keratinolytic action can be evaluated using feather broth and keratin medium and positive ability can be verified by media turbidity changes $[79,85]$. On the other hand, chitinolytic activity can be evaluated using powdered chitin agar [86] and pectinolytic activities can be evaluated with media containing pectin [87]. Both these deteriorative activities can be estimated and quantified [81]. Additionally, esterease action can be studied using Tributyrin agar and Tween 80 agar $[83,84,88]$. Their action can be detected by the development of clear zones (Tributyrin agar) or by the precipitation of insoluble salts and compounds (Tween 80) around colonies. As occurring with ligninolytic action, data regarding filamentous fungi fibroinase and keratinolytic action is still infrequent. Twenty-three species were found to be able of fibroinolytic activity, while more than twenty-five were found to have keratinolytic action. Again, Aspergillus and Penicillium species also dominate these biodeteriorative profiles (Tables 4 and 5). Moreover, various Alternaria species also displayed putative keratinolytic abilities. On the other hand, chitinolytic abilities have been identified for Aspergillus niger and Penicillium sp., while pectinolytic action has been identified in a slightly more diversified range of fungal genera and species (Aspergillus candidus, Mucor circinelloides, Penicillium echinulatum, Scopulariopsis koningii, Stachybotrys chartarum and Trichoderma hamatum) [81]. In parallel, fifty species have been identified as displaying estereolytic action, with a great dominance of Aspergillus and Penicillium species (Table 6). Understandably, the detection of these fungal species on crypt environments, human remains, buried materials, mummies, wax seals, textiles and clothes denotes a putative threat to these materials [3].

Table 4. Overview of fungal species for which isolates have been identified as displaying fibrinolytic abilities in biodeteriorative plate essays.

\begin{tabular}{|c|c|c|}
\hline Current Species Name & Original Study Focus & References \\
\hline \multirow{4}{*}{$\begin{array}{c}\text { Alternaria alternata (Fr.) Keissl. } \\
\text { Ascomycota Caval. -Sm. } \\
\text { Aspergillus caninus (Sigler, Deanna A. Sutton, Gibas, Summerb. and Iwen) Houbraken, } \\
\text { Tanney, Visagie and Samson }{ }^{1} \\
\text { Aspergillus cristatus Raper and Fennell }{ }^{2}\end{array}$} & Funeral accessories & [70] \\
\hline & Funeral clothes & [85] \\
\hline & Funeral clothes & [85] \\
\hline & Funeral clothes & [85] \\
\hline Aspergillus fumigatus Fresen. & Funeral clothes & [85] \\
\hline Aspergillus P. Micheli ex Haller & Funeral accessories & [80] \\
\hline Aspergillus puniceus Kwon-Chung and Fennell & Funeral clothes & [85] \\
\hline Aspergillus sydowii (Bainier and Sartory) Thom and Church & Funeral clothes & [85] \\
\hline Aspergillus tubingensis Mosseray & Funeral clothes & [85] \\
\hline Aspergillus versicolor (Vuill.) Tirab. & Funeral accessories & [80] \\
\hline Beauveria bassiana (Bals.-Criv.) Vuill. & Funeral clothes & [85] \\
\hline Myriodontium keratinophilum Samson and Polon. & Funeral clothes & [85] \\
\hline Penicillium brevicompactum Dierckx & Funeral clothes & [85] \\
\hline Penicillium commune Thom & Funeral accessories & [80] \\
\hline Penicillium crocicola T. Yamam. & Funeral clothes & [85] \\
\hline Penicillium crustosum Thom & Funeral clothes & [85] \\
\hline Penicillium expansum Link & Funeral clothes & [85] \\
\hline Penicillium granulatum Bainier & Funeral accessories & [80] \\
\hline Penicillium Link & Funeral accessories & [80] \\
\hline Penicillium roseopurpureum Dierckx & Funeral clothes & [85] \\
\hline Penicillium spinulosum Thom & Funeral clothes & [85] \\
\hline Sporobolomyces roseus Kluyver and C.B. Niel ${ }^{3}$ & Funeral accessories & [80] \\
\hline Xenochalara juniperi M.J. Wingf. and Crous & Funeral clothes & [85] \\
\hline
\end{tabular}

Previously identified as: ${ }^{1}$ Phialosimplex caninus; ${ }^{2}$ Eurotium cristatum; ${ }^{3}$ Sporidiobolus metaroseus. 
Table 5. Overview of fungal species for which isolates have been identified as displaying keratinolytic abilities in biodeteriorative plate essays.

\begin{tabular}{|c|c|c|}
\hline Current Species Name & Original Study Focus & References \\
\hline Alternaria consortialis (Thüm.) J.W. Groves and S. Hughes ${ }^{1}$ & Wooden art objects & [36] \\
\hline Arthrinium phaeospermum (Corda) M.B. Ellis & Wooden art objects & [36] \\
\hline Aspergillus amstelodami (L. Mangin) Thom and Church ${ }^{2}$ & Wooden art objects & [36] \\
\hline Aspergillus awamori Nakaz. & Wooden art objects & [36] \\
\hline Aspergillus fischeri Wehmer ${ }^{3}$ & Wooden art objects & [36] \\
\hline Aspergillus flavus Link & Wooden art objects & [36] \\
\hline Aspergillus fumigatus Fresen. & Wooden art objects & [36] \\
\hline Aspergillus niger Tiegh & Wooden art objects & [36] \\
\hline Aspergillus terreus Thom & Wooden art objects and air & {$[36,69]$} \\
\hline Aspergillus ustus (Bainier) Thom and Church & Wooden art objects & {$[36]$} \\
\hline Aspergillus versicolor (Vuill.) Tirab. & Wooden art objects & [36] \\
\hline Beauveria bassiana (Bals.-Criv.) Vuill. & Wooden art objects & [36] \\
\hline Chaetomium elatum Kunze & Wooden art objects & [36] \\
\hline Chaetomium globosum Kunze & Wooden art objects and air & {$[36,69]$} \\
\hline Cladosporium cladosporioides (Fresen.) G.A. de Vries & Wooden objects and air & [69] \\
\hline Cladosporium herbarum (Pers.) Link & Wooden art objects & [36] \\
\hline Cladosporium Link & Wooden art objects & [36] \\
\hline Hypochnicium J. Erikss. & Wooden materials & {$[61]$} \\
\hline "Neocosmospora" solani (Mart.) L. Lombard and Crous 4 & Wooden materials & [70] \\
\hline Penicillium chrysogenum Thom & Wooden art objects & [36] \\
\hline Penicillium expansum Link & Wooden art objects & [36] \\
\hline Penicillium glabrum (Wehmer) Westling & Wooden art objects & [36] \\
\hline Penicillium herquei Bainier and Sartory & Wooden art objects & [36] \\
\hline Penicillium Link & Wooden art objects & [36] \\
\hline Penicillium sacculum E. Dale & Wooden art objects & [36] \\
\hline Pseudogymnoascus pannorum (Link) Minnis and D.L. Lindner ${ }^{5}$ & Wooden art objects & [36] \\
\hline Trichoderma viride Pers. & Wooden art objects & [36] \\
\hline
\end{tabular}

Previously identified as: ${ }^{1}$ Alternaria consortiale; ${ }^{2}$ Eurotium amstelodami ${ }^{3}$ Neosartorya fischeri; ${ }^{4}$ Fusarium solani; ${ }^{5}$ Chrysosporium pannorum.

Table 6. Overview of fungal species for which isolates have been identified as displaying estereolytic abilities in biodeteriorative plate essays.

\begin{tabular}{|c|c|c|}
\hline Current Species Name & Original Study Focus & References \\
\hline Acrodontium salmoneum de Hoog & Wax seal & [84] \\
\hline Agaricaceae Chevall. & Statue and air & [89] \\
\hline Alternaria Nees & Statue, stone and air & {$[89,90]$} \\
\hline Alternaria tenuissima (Kunze) Wiltshire & Statue and air & [89] \\
\hline Aspergillus amstelodami (L. Mangin) Thom and Church ${ }^{1}$ & Stone & [90] \\
\hline Aspergillus caespitosus Raper and Thom & Air, textiles and human remains & [83] \\
\hline Aspergillus calidoustus Varga, Houbraken and Samson & Air, textiles and human remains & [83] \\
\hline Aspergillus candidus Link & Air, textiles and human remains & [83] \\
\hline Aspergillus clavatus Desm. & Textiles & {$[82]$} \\
\hline Aspergillus fischeri Wehmer ${ }^{2}$ & Air, textiles and human remains & [83] \\
\hline Asperoillus flavus Link & Stone & [90] \\
\hline Aspergillus fumigatus Fresen. & Air, statues, textiles and human remains & {$[83,89]$} \\
\hline Aspergillus niger Tiegh & Stone & [90] \\
\hline Aspergillus repens (Corda) Sacc. ${ }^{3}$ & Air, textiles and human remains & [83] \\
\hline Aspergillus sydowii (Bainier and Sartory) Thom and Church & Air, textiles and human remains & [83] \\
\hline Aspergillus terreus Thom & Air, textiles and human remains & [83] \\
\hline Aspergillus ustus (Bainier) Thom and Church & Air, textiles and human remains & [83] \\
\hline Aspergillus venenatus Jurjević, S.W. Peterson and B.W. Horn & Air, textiles and human remains & [83] \\
\hline Aspergillus versicolor (Vuill.) Tirab. & Air, statues, textiles and human remains & {$[83,89]$} \\
\hline Aspergillus westerdijkiae Frisvad and Samson & Air, textiles and human remains & [83] \\
\hline Aureobasidium pullulans (de Bary and Löwenthal) G. Arnaud & Wax seal & [84] \\
\hline Bulleromyces albus Boekhout and Á. Fonseca 4 & Wax seal & [84] \\
\hline Cladosporium aggregatocicatricatum Bensch, Crous and U. Braun & Wax seal & [84] \\
\hline Cladosporium Link & Air, textiles and human remains & [83] \\
\hline Cladosporium macrocarpum Preuss & Statue and air & [89] \\
\hline Cladosporium tenuissimum Cooke & Textiles & [82] \\
\hline
\end{tabular}


Table 6. Cont.

\begin{tabular}{|c|c|c|}
\hline Current Species Name & Original Study Focus & References \\
\hline Coprinellus xanthothrix (Romagn.) Vilgalys, Hopple and Jacq. Johnson & Air, textiles and human remains & [83] \\
\hline Coprinopsis cinerea (Schaeff.) Redhead, Vilgalys and Moncalvo & Statue and air & [89] \\
\hline Curvularia Boedijn & Stone & [90] \\
\hline Fusarium Link & Stone & [90] \\
\hline Fusarium sporotrichioides Sherb. & Statue and air & [89] \\
\hline Hypoxylon fragiforme (Pers.) J. Kickx f. & Textiles & [82] \\
\hline Microascus brevicaulis S.P. Abbott ${ }^{5}$ & Air, textiles and human remains & [83] \\
\hline Nigrospora oryzae (Berk. and Broome) Petch & Air, textiles and human remains & [83] \\
\hline Penicillium chrysogenum Thom & Air, textiles and human remains & {$[82,83]$} \\
\hline Penicillium commune Thom & Air, textiles and human remains & [83] \\
\hline Penicillium corylophilum Dierckx & Textiles, statue and air & {$[82,89]$} \\
\hline Penicillium crustosum Thom & Air, statues, textiles and human remains & {$[83,89]$} \\
\hline Penicillium griseofulvum Dierckx & Air, textiles and human remains & [83] \\
\hline Penicillium hordei Stolk & Air, textiles and human remains & [83] \\
\hline Penicillium Link & Statue and air & {$[89]$} \\
\hline Penicillium polonicum K.W. Zaleski & Air, textiles and human remains & [83] \\
\hline Pseudoscopulariopsis hibernica (A. Mangan) Sand. -Den., Gené and Cano ${ }^{6}$ & Air, textiles and human remains & [83] \\
\hline Rhizopus arrhizus A. Fisch. ${ }^{7}$ & Air, textiles and human remains & [83] \\
\hline Rhizopus microsporus Tiegh. & Air, textils, human remains, statue and air & {$[83,89]$} \\
\hline Rhizopus stolonifer (Ehrenb.) Vuill. & Air, textiles and human remains & [83] \\
\hline Sordaria fimicola (Roberge ex Desm.) Ces. and De Not. & Statue and air & [89] \\
\hline $\begin{array}{l}\text { Talaromyces purpureogenus Samson, N. Yilmaz, Houbraken, Spierenb., } \\
\text { Seifert, Peterson, Varga and Frisvad }\end{array}$ & Stone & [90] \\
\hline Thyronectria austroamericana (Speg.) Seeler 8 & Statue and air & [89] \\
\hline Trichoderma paraviridescens Jaklitsch, Samuels and Voglmayr & Statue and air & [89] \\
\hline
\end{tabular}

Previously identified as: ${ }^{1}$ Eurotium amsteldomi; ${ }^{2}$ Neosartorya fischeri; ${ }^{3}$ Eurotium repens; ${ }^{4}$ Bullera alba $;{ }^{5}$ Scopulariopsis brevicaulis; ${ }^{6}$ Scopulariopsis hibernica; ${ }^{7}$ Rhizopus oryzae; ${ }^{8}$ Pleonectria austroamericana.

Fungal lipolytic action can have an important impact on the biodeterioration of parchment and leather related materials [91]. Fungi can attack lipids and take advantage of fatty materials as a mean to obtain carbon (while also contributing to the material deterioration) [92]. Fungal lipolytic ability characterization in cultural heritage scenarios has been helpful to study the biodeteriorative contribution of isolates retrieved from air, textiles, human remains, wax seals, albumen photographical materials, statues, wooden organs and pipes $[83,84,89,93,94]$. Lipolytic ability screening can be mainly conducted using Spirit Blue agar and Nile blue, and the positive action can be identified by the development of a halo around the colonies, after a period of incubation [89]. Circa sixty species were found to be able of lipolytic action (Table 7). As similarly verified in other deteriorative analyses, Aspergillus and Penicillium species are still predominant in these profiles. The detection of these fungal species on materials rich in fatty compounds, such as wax seals and photographic materials should be considered putatively hazardous.

Table 7. Overview of fungal species for which isolates have been identified as displaying lipolytic abilities in biodeteriorative plate essays.

\begin{tabular}{|c|c|c|}
\hline Current Species Name & Original Study Focus & References \\
\hline Acrodontium salmoneum de Hoog & Wax seal & [84] \\
\hline Acrostalagmus luteoalbus (Link) Zare, W. Gams and Schroers & Albumen photographic materials & [93] \\
\hline Agaricaceae Chevall. & Statue and air & [89] \\
\hline Alternaria mali Roberts & Wooden organ and pipes & [94] \\
\hline Alternaria Nees & Statue and air & [89] \\
\hline Alternaria tenuissima (Kunze) Wiltshire & Statue and air & [89] \\
\hline Aspergillus amstelodami (L. Mangin) Thom and Church ${ }^{1}$ & $\begin{array}{c}\text { Air, textiles, human remains, wooden } \\
\text { organ and pipes }\end{array}$ & {$[83,94]$} \\
\hline Aspergillus caespitosus Raper and Thom & Air, textiles and human remains & [83] \\
\hline Aspergillus calidoustus Varga, Houbraken and Samson & Air, textiles and human remains & [83] \\
\hline Aspergillus candidus Link & Air, textiles and human remains & [83] \\
\hline Aspergillus cristatus Raper and Fennell ${ }^{2}$ & Wooden organ and pipes & [94] \\
\hline
\end{tabular}


Table 7. Cont.

\begin{tabular}{|c|c|c|}
\hline Current Species Name & Original Study Focus & References \\
\hline Aspergillus fischeri Wehmer ${ }^{3}$ & $\begin{array}{l}\text { Air, textiles, statues and human } \\
\text { remains }\end{array}$ & {$[89,93]$} \\
\hline Aspergillus fumigatus Fresen. & $\begin{array}{l}\text { Air, textiles, statues and human } \\
\text { remains }\end{array}$ & {$[83,89]$} \\
\hline Aspergillus repens (Corda) Sacc. ${ }^{4}$ & Air, textiles and human remains & [83] \\
\hline Aspergillus sydowii (Bainier and Sartory) Thom and Church & $\begin{array}{l}\text { Air, textiles, human remains, wooden } \\
\text { organ and pipes }\end{array}$ & {$[83,94]$} \\
\hline Aspergillus terreus Thom & Air, textiles and human remains & [83] \\
\hline Aspergillus ustus (Bainier) Thom and Church & Air, textiles and human remains & [83] \\
\hline Aspergillus venenatus Jurjević, S.W. Peterson and B.W. Horn & Air, textiles and human remains & [83] \\
\hline Aspergillus versicolor (Vuill.) Tirab & $\begin{array}{c}\text { Air, textiles, human remains, albumen } \\
\text { photographic materials, wooden organ } \\
\text { and pipes }\end{array}$ & {$[83,89,93,94]$} \\
\hline Aspergillus westerdijkiae Frisvad and Samson & Air, textiles and human remains & [83] \\
\hline Aureobasidium pullulans (de Bary and Löwenthal) G. Arnaud & $\begin{array}{l}\text { Wax seal } \\
\text { Albumen }\end{array}$ & {$[84]$} \\
\hline $\begin{array}{l}\text { Bjerkandera adusta (Willd.) P. Karst. } \\
\text { Bulleromuces }\end{array}$ & $\begin{array}{c}\text { Albumen photographic materials } \\
\text { Wax seal }\end{array}$ & {$[83]$} \\
\hline Bulleromyces albus Boekhout and Á. Fonseca ${ }^{5}$ & Wax seal & [84] \\
\hline Chaetomium elatum Kunze & Albumen photographic materials & [83] \\
\hline Cladosporium aggregatocicatricatum Bensch, Crous and U. Braun & Wax seal & [84] \\
\hline Cladosporium cladosporioides (Fresen.) G.A. de Vries & Wooden organ and pipes & [94] \\
\hline Cladosporium Link & $\begin{array}{l}\text { Air, textiles, human remains and } \\
\text { Etruscan hypogeal tombs }\end{array}$ & {$[44,83]$} \\
\hline \multirow{2}{*}{$\begin{array}{c}\text { Cladosporium macrocarpum Preuss } \\
\text { Coprinellus xanthothrix (Romagn.) Vilgalys, Hopple and Jacq. } \\
\text { Johnson }\end{array}$} & Statue and air & [89] \\
\hline & Air, textiles and human remains & [83] \\
\hline Coprinopsis cinerea (Schaeff.) Redhead, Vilgalys and Moncalvo & Statue and air & [89] \\
\hline Cyphellophora G.A. de Vries & Etruscan hypogeal tombs & [44] \\
\hline Cyphellophora olivacea (W. Gams) Réblová \& Unter. & Etruscan hypogeal tombs & [44] \\
\hline Epicoccum nigrum Link & Wooden organ and pipes & [94] \\
\hline Exophiala angulospora Iwatsu, Udagawa \& T. Takase & Etruscan hypogeal tombs & [44] \\
\hline Exophiala J.W. Carmich. & Etruscan hypogeal tombs & [44] \\
\hline Fusarium sporotrichioides Sherb. & Statue and air & [89] \\
\hline Microascus brevicaulis S.P. Abbott ${ }^{6}$ & Air, textiles and human remains & [83] \\
\hline Mucor plumbeus Bonord. & Albumen photographic materials & [93] \\
\hline Nigrospora oryzae (Berk. and Broome) Petch & Air, textiles and human remains & [83] \\
\hline Paecilomyces maximus C. Ram & Wooden organ and pipes & [94] \\
\hline Penicillium chrysogenum Thom & Air, textiles and human remains & [83] \\
\hline Penicillium commune Thom & Air, textiles and human remains & {$[83]$} \\
\hline Penicillium corylophilum Dierckx & Statue and air & [89] \\
\hline Penicillium crustosum Thom & $\begin{array}{c}\text { Air, textiles, statues and human } \\
\text { remains }\end{array}$ & {$[83,89]$} \\
\hline Penicillium digitatum (Pers.) Sacc. & Air, textiles and human remains & [83] \\
\hline Penicillium griseofulvum Dierckx & Air, textiles and human remains & [83] \\
\hline Penicillium hordei Stolk & Air, textiles and human remains & [83] \\
\hline Penicillium Link & $\begin{array}{l}\text { Albumen photographic materials, } \\
\text { statues and air }\end{array}$ & {$[89,93]$} \\
\hline Penicillium polonicum K.W. Zaleski & Air, textiles and human remains & [83] \\
\hline Penicillium thomii Maire & Albumen photographic materials & [93] \\
\hline Phlebia Fr. & Albumen photographic materials & [93] \\
\hline \multirow{2}{*}{$\begin{array}{c}\text { Pleosporales Luttr. ex M.E. Barr } \\
\text { Pseudoscopulariopsis hibernica (A. Mangan) Sand. -Den., Gené } \\
\text { and Cano }{ }^{7}\end{array}$} & Albumen photographic materials & [93] \\
\hline & Air, textiles and human remains & [83] \\
\hline Rhizopus arrhizus A. Fisch. ${ }^{8}$ & Air, textiles and human remains & [83] \\
\hline Rhizopus microsporus Tiegh. & $\begin{array}{l}\text { Air, textiles, human remains, statues } \\
\text { and air }\end{array}$ & {$[83,89]$} \\
\hline Rhizopus stolonifer (Ehrenb.) Vuill. & Air, textiles and human remains & [83] \\
\hline Sordaria fimicola (Roberge ex Desm.) Ces. and De Not. & Statue and air & [89] \\
\hline Talaromyces flavus (Klöcker) Stolk and Samson & Air, textiles and human remains & [83] \\
\hline Thyronectria austroamericana (Speg.) Seeler ${ }^{9}$ & Statue and air & [89] \\
\hline Trichoderma harzianum Rifai & Air, textiles and human remains & [83] \\
\hline Trichoderma paraviridescens Jaklitsch, Samuels and Voglmayr & Statue and air & [89] \\
\hline Trichothecium roseum (Pers.) Link & Albumen photographic materials & [93] \\
\hline
\end{tabular}

Previously identified as: ${ }^{1}$ Eurotium amstelodami; ${ }^{2}$ Eurotium cristatum $;{ }^{3}$ Neosartorya fischeri; ${ }^{4}$ Eurotium repens; ${ }^{5}$ Bullera alba; ${ }^{6}$ Scopulariopsis brevicaulis; ${ }^{7}$ Scopulariopsis hibernica; ${ }^{8}$ Rhizopus oryzae; ${ }^{9}$ Pleonectria austroamericana. 
Fungal proteolytic action can contribute to the biodeterioration of proteinaceous materials, such is the case of artistic natural binders. In addition, some conservation approaches also employ similar materials that can be targeted by microbial biodeterioration [38]. Fungal proteolytic ability characterization in cultural heritage scenarios has been helpful to study the biodeteriorative contribution of isolates retrieved from air, funeral clothes and accessories, graphic documents, materials present in libraries and museums, frescoes, textiles, human remains, mummies, mural paintings, cinematographic films, wax seals, paper, parchment, wooden organs and pipes [28,38,41,78-80,83-85,93,95-100]. Proteolytic ability screening can be mainly conducted using Gelatin agar (R2A-Gel), Casein agar (CN), Milk Nutrient agar (MilkNA) and media containing rabbit glue [78,94,96]. After a period of incubation, positive proteolytic ability can be detected by flooding of agar plates with $10 \%$ tannin solution and the visualization of the formed hydrolysis zones [101]. Over one hundred and thirty species have been found to be able of promoting protein attack (Table 8). As similarly verified in other enzymatic activities, Aspergillus and Penicillium species also dominate this biodeteriorative profile. Nonetheless, a significant number of species from genera Alternaria, Cladosporium and Talaromyces displaying these characteristics can also be confirmed. Detection of these fungal species on proteinaceous materials will putatively result in their accentuated biodeterioration.

Table 8. Overview of fungal species for which isolates have been identified as displaying proteolytic abilities in biodeteriorative plate essays.

\begin{tabular}{|c|c|c|}
\hline Current Species Name & Original Study Focus & References \\
\hline Acrodontium salmoneum de Hoog & Wax seal & [84] \\
\hline Acrostalagmus luteoalbus (Link) Zare, W. Gams and Schroers & Albumen photographic materials & [93] \\
\hline Actinomucor elegans (Eidam) C.R. Benjamin and Hesseltine & Mural paintings & [41] \\
\hline Alternaria alternata (Fr.) Keissl. & $\begin{array}{l}\text { Air, mural paintings, mummies, funeral accessories, } \\
\text { cinematographic films }\end{array}$ & {$[41,79,80,95,98]$} \\
\hline Alternaria mali Roberts & Mummies, wooden organ and pipes & {$[79,94]$} \\
\hline Alternaria Nees & Mummies and funeral clothes & {$[79,85]$} \\
\hline Alternaria solani Sorauer & Mummies & [79] \\
\hline Alternaria tenuiss & Mummies & [79] \\
\hline Ascomyce & Funeral clothes & [85] \\
\hline Aspergillus alliaceus Thom and Church & Air and graphic documents & {$[98,100]$} \\
\hline Aspergillus amstelodami (L. Mangin) Thom and Church & Air, wooden organ and pipes & {$[28,94]$} \\
\hline Aspergillus aureolatus Munt.-Cvetk. and Bata & Frescoes and air & [38] \\
\hline Aspergillus auricomus (Guég.) Saito & Air and graphic documents & [100] \\
\hline Aspergillus caespitosus Raper and Thom & Air, textiles and human remains & [83] \\
\hline Aspergillus candidus Link & $\begin{array}{c}\text { Air, graphic documents, photographs, maps, textiles } \\
\text { and human remains }\end{array}$ & {$[83,84,98-100]$} \\
\hline $\begin{array}{l}\text { Aspergillus caninus (Sigler, Deanna A. Sutton, Gibas, Summerb. } \\
\text { and Iwen) Houbraken, Tanney, Visagie and Samson }{ }^{1}\end{array}$ & Funeral clothes & [85] \\
\hline Aspergillus chevalieri (L. Mangin) Thom and Church ${ }^{2}$ & $\begin{array}{l}\text { Air, graphic documents, photographs, maps and } \\
\text { materials present in libraries and museums }\end{array}$ & {$[96,99,100]$} \\
\hline $\begin{array}{l}\text { Aspergillus creber Jurjević, S.W. Peterson and B.W. Horn } \\
\text { Aspergillus cristatus Raper and Fennell }{ }^{3}\end{array}$ & $\begin{array}{c}\text { Air } \\
\text { Funeral clothes, wooden organ and pipes }\end{array}$ & {$\left[\begin{array}{c}{[28]} \\
{[85,94]}\end{array}\right.$} \\
\hline Aspergillus europaeus Hubka, A. Nováková, Samson, & Frescoes and air & {$[28,38]$} \\
\hline $\begin{array}{l}\text { Houbraken, Frisvad and M. Kolařík } \\
\text { Aspergillus flavipes (Bainier and R. Sartory) Thom and Church }\end{array}$ & Air & {$[98]$} \\
\hline Aspergillus flavus Link $^{4}$ & $\begin{array}{l}\text { Air, frescoes, mummies, photographs, maps and } \\
\text { materials present in libraries and museums }\end{array}$ & {$[38,47,78,79,96,99,100]$} \\
\hline Aspergillus fumigatus Fresen. & $\begin{array}{l}\text { Air, materials present in libraries and museums, } \\
\text { paper and parchment and funeral clothes }\end{array}$ & {$[85,96,97]$} \\
\hline $\begin{array}{l}\text { Aspergillus japonicus Saito } \\
\text { Aspergillus jensenii Jurjević, S.W. Peterson and B.W. Horn }\end{array}$ & $\begin{array}{l}\text { Air and graphic documents } \\
\text { Air }\end{array}$ & {$\left[\begin{array}{l}{[100]} \\
{[28]}\end{array}\right.$} \\
\hline Aspergillus nidulans (Eidam) G. Winter & $\begin{array}{l}\text { Air, materials present in libraries and museum, } \\
\text { mural paintings and cinematographic films }\end{array}$ & {$[41,95,96]$} \\
\hline Aspergillus niger Tiegh & $\begin{array}{l}\text { Frescoes, air, textiles, human remains, photographs, } \\
\text { maps, paper and parchment, graphic documents, } \\
\text { materials present in libraries and museums }\end{array}$ & {$[38,73,96-100]$} \\
\hline Aspergillus ostianus Wehme & Frescoes, air and graphic documents & {$[38,100]$} \\
\hline Aspergillus P. Micheli ex Haller ${ }^{5}$ & Air and mural paintings & {$[41,47]$} \\
\hline ioides Speg. & Air and graphic documents & [100] \\
\hline erans G. Sm. & Air & [28] \\
\hline Aspergillus protuberus Munt. -Cvetk. & Air & [28] \\
\hline Aspergillus pseudodeflectus Samson and Mouch. & Funeral clothes & {$[85]$} \\
\hline Aspergillus puniceus Kwon-Chung and Fennell & Funeral clothes & [85] \\
\hline
\end{tabular}


Table 8. Cont.

Current Species Name
Aspergillus sydowii (Bainier and Sartory) Thom and Church
Aspergillus tabacinus Nakaz., Y. Takeda, Simo and A. Watan.
Aspergillus tamarii Kita
Aspergillus terreus Thom
Aspergillus tubingensis Mosseray
Aspergillus unilateralis Thrower
Aspergillus unguis (Émile-Weill and L. Gaudin) Thom and Raper
Aspergillus ustus (Bainier) Thom and Church
Aspergillus versicolor (Vuill.) Tirab
Aspergillus westerdijkiae Frisvad and Samson
Beauveria bassiana (Bals.-Criv.) Vuill.
Bjerkandera adusta (Willd.) P. Karst.
Aureobasidium pullulans (de Bary and Löwenthal G. Weing
Botryotrichum murorum (Corda) X. Wei Wang and Samson ${ }^{6}$
Bulleromyces albus Boekhout and Á. Fonseca ${ }^{7}$
Chaetomium ancistrocladum Udagawa and Cain
Chaetomium elatum Kunze
Chaetomium globosum Kunze
Cladosporium aggregatocicatricatum Bensch, Crous and U. Braun

Cladosporium cladosporioides (Fresen.)

Cladosporium cucumerinum Ellis and Arthur

Cladosporium herbarum (Pers.) Link ${ }^{8}$ Cladosporium Link

Cladosporium macrocarpum Preuss

Cladosporium oxysporum Berk. and M.A. Curtis

Cladosporium pseudocladosporioides Bensch, Crous and U. Braun Cladosporium sphaerospermum Penz.

Cladosporium tenuissimum Cooke

Cladosporium uredinicola Speg.

Coprinellus xanthothrix (Romagn.) Vilgalys, Hopple and Jacq. Johnson

Curvularia australiensis (Bugnic. ex M.B. Ellis) Manamgoda, L.

$$
\begin{gathered}
\text { Cai and K.D. Hyde } \\
\text { Curvularia }{ }^{9} \text { Boedijn }
\end{gathered}
$$

Curvularia pallescens Boedijn

Cyphellophora G.A. de Vries

Didymella glomerata (Corda) Qian Chen and L. Cai 10

Dipodascus Lagerh. 11 Epicoccum Link

Epicoccum nigrum Link

Fusarium chlamydosporum Wollenw. and Reinking Fusarium Link

Fusarium oxysporum Schltdl.

Hormodendrum pyri W.H. English

Leptosphaeria avenaria G.F. Weber 12

Meyerozyma guilliermondii (Wick.) Kurtzman and M. Suzuki ${ }^{13}$

Microascus brevicaulis S.P. Abbott ${ }^{14}$

Mucor racemosus Bull.

Mucor spinosus Schrank

Myriodontium keratinophilum Samson and Polon.

Nigrospora oryzae (Berk. and Broome) Petch ${ }^{15}$

Paecilomyces maximus C. Ram ${ }^{16}$

Paecilomyces variotii Bainier

Penicillium aurantiogriseum Dierckx Penicillium bilaiae Chalab.

Penicillium brevicompactum Dierckx

Penicillium camemberti Thom

Penicillium canescens Sopp

Penicillium chrysogenum Thom

Penicillium citreonigrum Dierckx Penicillium citrinum Thom

Penicillium commune Thom

Penicillium crocicola T. Yamam. Penicillium crustosum Thom
Air, mural paintings, funeral clothes, wooden organ and pipes and materials present in libraries and museums

Air and materials present in libraries and museums Air and materials present in libraries and museums Air and materials present in libraries and museums Funeral clothes

Air and graphic documents

Air and graphic documents Cinematographic films

Air, mural paintings, frescoes, textiles, human remains, funeral accessories, cinematographic materials, wooden organ and pipes and materials present in libraries and museums Air, textiles and human remains Wax seal and mummies Funeral clothes

Frescoes, air albumen photographic materials Frescoes and air Wax seal

Frescoes and air

Albumen photographic materials Mural paintings Wax seal

Mural paintings, frescoes, air, mummies, graphic documents, maps, photographs, cinematographic films, wooden organ and pipes Mural paintings

Mummies and mural painitngs

Etruscan hypogeal tombs, air and mummies Air and frescoes
Frescoes and air Mummies

Mummies and mural paintings Mural paintings

Mummies, frescoes and air

Air, textiles and human remains

Air and graphic documents Air

Air and graphic documents

Etruscan hypogeal tombs

Cinematographic films Air

Air and graphic documents Mummies, wooden organ and pipes Mural paintings

Air and graphic documents Air Wax seal frescoes and air

$$
\text { Air }
$$

Air, textiles and human remains Cinematographic films, paper and parchment Paper and parchment Funeral clothes

Air, textiles and human remains

Wooden organ and pipes Air

Mural paintings

Frescoes and air

Air and funeral clothes

Paper and parchment

Air and graphic documents

Air, mural paintings, textiles, human remains, mummies, paper, photographs, maps, parchment, graphic documents and cinematographic films

$$
\text { Air }
$$

Air, photographs, maps and graphic documents

Air, textiles, human remains, mural paintings, funeral accessories and clothes, paper and parchment Funeral clothes

Funeral clothes, wooden organ and pipes
References

$[28,41,85,94,96]$

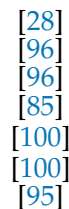

$[28,38,41,78,80,83,94-$

96]

$[83]$

$[79,84]$
$[85]$

$[85]$

[38]

[84]

[38]

[93]

[84]

[38,41,79,94,95,99,100]

[41,81]

$[44,47,79]$

[78]

[38]

[41,79]

[41]

[83]

[100]

[47]

[98,100]

[44]

[95]

[47]

[100]

$[79,94]$

[41]

$[57,100]$

[98]

[38]

[98]

[83]

[95,97]

[97]

$[83,98]$

[94]

[98]

[38]

[28,85]

[97]

[100]

$[28,41,79,83,95,97,99$,

100]

[28]

$[28,99,100]$

[41,80,83,85,87]

[85]

$[85,94]$ 
Table 8. Cont

\begin{tabular}{|c|c|c|}
\hline Current Species Name & Original Study Focus & References \\
\hline Penicillium decumbens Thom & Air, paper and parchment & {$[28,97]$} \\
\hline Penicillium digitatum (Pers.) Sacc. & Air, textiles and human remains & {$[28,83]$} \\
\hline Penicillium expansum Link & Air, mummies and funeral clothes & {$[28,79,85]$} \\
\hline Penicillium glabrum (Wehmer) Westling & Air & [28] \\
\hline Penicillium granulatum Bainier & Funeral accessories & [80] \\
\hline Penicillium griseofulvum Dierckx & $\begin{array}{c}\text { Air, frescoes, graphic documents, textiles and human } \\
\text { remains }\end{array}$ & {$[38,83,100]$} \\
\hline $\begin{array}{l}\text { Penicillium hordei Stolk } \\
\text { Penicillium janczewskii K.W. Zaleski }\end{array}$ & $\begin{array}{l}\text { Air, textiles and human remains } \\
\text { Air, maps, photographs and graphic documents }\end{array}$ & $\begin{array}{c}{[83]} \\
{[99,100]}\end{array}$ \\
\hline Penicillium Link & $\begin{array}{c}\text { Air, frescoes, albumen photographic materials and } \\
\text { funeral accessories }\end{array}$ & {$[57,78,80,93]$} \\
\hline Penicillium olsonii Bainier and Sartory & Mural paintings & [41] \\
\hline Penicillium polonicum K.W. Zaleski & Air, textiles, mural paintings and human remains & {$[41,83]$} \\
\hline Penicillium raistrickii G. Sm. & Mummies & [79] \\
\hline Penicillium roseopurpureum Dierckx & Mummies and funeral clothes & {$[79,85]$} \\
\hline Penicillium simplicissimum (Oudem.) Thom ${ }^{17}$ & Air, maps, photographs and graphic documents & {$[99,100]$} \\
\hline Penicillium solitum Westling & Air & [28] \\
\hline Penicillium spinulosum Thom & Funeral clothes & {$[85]$} \\
\hline $\begin{array}{l}\text { Penicillium viridicatum } \\
\text { Westling }\end{array}$ & Air, paper and parchment & {$[28,97]$} \\
\hline Penicillium waksmanii K.W. Zaleski & Air and graphic documents & [100] \\
\hline Pestalotia De Not. & Air and graphic documents & {$[100]$} \\
\hline Phoma herbarum Westend. & Paper and parchment & [97] \\
\hline Pleurotus ostreatus (Jacq.) P. Kumm. ${ }^{18}$ & Albumen photographic materials & [93] \\
\hline Pseudozyma prolifica Bandoni & Mural paintings & [31] \\
\hline Rhizopus microsporus Tiegh. ${ }^{19}$ & Air, textiles and human remains & [83] \\
\hline Sarocladium W. Gams and D. Hawksw. ${ }^{20}$ & $\begin{array}{l}\text { Air, graphic documents and albumen photographic } \\
\text { materials }\end{array}$ & {$[93,98,100]$} \\
\hline Sporobolomyces roseus Kluyver and C.B. Niel ${ }^{21}$ & Funeral Accessories & [80] \\
\hline Stemphylium vesicarium (Wallr.) E.G. Simmons & Mummies & [79] \\
\hline $\begin{array}{c}\text { Talaromyces aculeatus (Raper and Fennell) Samson, N. Yilmaz, } \\
\text { Frisvad and Seifert }\end{array}$ & Mural paintings & [41] \\
\hline $\begin{array}{c}\text { Talaromyces rugulosus (Thom) Samson, N. Yilmaz, Frisvad } \\
\text { and Seifert }\end{array}$ & Wooden organ and pipes & [94] \\
\hline Talaromyces sayulitensis Visagie, N. Yilmaz, Seifert and Samson & Air & [28] \\
\hline Trichoderma harzianum Rifai & Air, textiles and human remains & [83] \\
\hline Trichoderma longibrachiatum Rifai & Cinematographic films & [95] \\
\hline Trichoderma viride Pers. & Air & [98] \\
\hline Trichothecium roseum (Pers.) Link & Albumen photographic materials & [93] \\
\hline Xenochalara juniperi M.J. Wingf. and Crous & Funeral clothes & [85] \\
\hline
\end{tabular}

Previously identified as: ${ }^{1}$ Phialosimplex caninus; ${ }^{2}$ Eurotium chevalieri ${ }^{3}$ Eurotium cristatum $;{ }^{4}$ Aspergillus oryzae; ${ }^{5}$ Emericella sp.; ${ }^{6}$ Chaetomium murorum; ${ }^{7}$ Bullera alba; ${ }^{8}$ Cladosporium herbarium; ${ }^{9}$ Bipolaris australiensis; ${ }^{10}$ Phoma glomerata; ${ }^{11}$ Geotrichum sp.; ${ }^{2}$ Phaeosphaeria avenaria; ${ }^{13}$ Candida guilliermondii; ${ }^{14}$ Scopulariopsis brevicaulis; ${ }^{15}$ Nigrospora sphaerica; ${ }^{16}$ Paecilomyces formosus; ${ }^{17}$ Penicillium janthinellum; ${ }^{18}$ Pleurotus pulmonarius; ${ }^{19}$ Rhizopus microsporus var. oligosporus; ${ }^{20}$ Cephalosporium sp.; ${ }^{21}$ Sporidiobolus metaroseus.

Fungal cellulolytic action is known to contribute to the biodeterioration of paper, canvas oil paintings, binders and photographic materials [3]. Moreover, cellulolytic action abilities characterization in cultural heritage scenarios has been helpful to study the biodeteriorative contribution of isolates retrieved from air, albumen photographic materials, mummies, funeral accessories, wooden art objects, organs and pipes, wax seals, graphic documents, stone, drawings, lithographs, paintings, textiles, human remains, maps, photographs, paper and other materials present in libraries and museums [28,36,69,79,81-83,96-100,102-105]. Cellulolytic ability screening can be conducted using Czapek-Dox agar supplemented with hydroxyethyl cellulose [69], Congo Red agar [79], Mandels and Reese medium with carboxymethyl cellulose (CMC) [106] or media containing sterilized filter paper [47]. Positive evaluation of cellulolytic ability can be assessed by the visualization of hydrolyzed areas or after congo red application and treatment. Over one hundred and fifty fungal species have been found to have cellulolytic abilities (Table 9). The great majority of species belonged to genera Alternaria, Aspergillus, Chaetomium, Cladosporium, Penicillium and Talaromyces. As such, detection of these fungal species on cellulolytic materials including paper, paintings and photographic materials, should be perceived as putatively threatening from a biodeterioration standpoint. 
Table 9. Overview of fungal species for which isolates have been identified as displaying cellulolytic abilities in biodeteriorative plate essays.

\begin{tabular}{|c|c|c|}
\hline Current Species Name & Original Study Focus & References \\
\hline Acremonium alabamense Morgan-Jones & Air & [98] \\
\hline Acrostalagmus luteoalbus (Link) Zare, W. Gams and Schroers & Albumen photographic materials & [93] \\
\hline Alternaria alternata (Fr.) Keissl. & Air, mummies and funeral accessories & {$[79,80,98]$} \\
\hline Alternaria chartarum Preuss ${ }^{1}$ & Mummies & [81] \\
\hline Alternaria consortialis (Thüm.) J.W. Groves and S. Hughes ${ }^{2}$ & Wooden art objects & [36] \\
\hline Alternaria mali Roberts & Mummies, wooden organ and pipes & {$[79,94]$} \\
\hline Alternaria Nees & $\begin{array}{c}\text { Air and graphic documents, stone and } \\
\text { mummies }\end{array}$ & {$[47,79,90,91]$} \\
\hline Alternaria solani Sorauer & Mummies & [79] \\
\hline Alternaria tenuissima (Kunze) Wiltshire & Air and mummies & {$[79,102]$} \\
\hline Arthrinium arundinis (Corda) Dyko and B. Sutton & Drawings and lithographs & [94] \\
\hline Arthrinium phaeospermum (Corda) M.B. Ellis & Wooden art objects & [36] \\
\hline Ascomycota Caval. -Sm. & Funeral clothes and oainting & {$[85,103]$} \\
\hline Ascotricha Berk. & Painting & [103] \\
\hline Ascotricha chartarum Berk. & Painting & [103] \\
\hline Aspergillus alliaceus Thom and Church & Air and graphic documents & {$[98,100]$} \\
\hline Aspergillus amstelodami (L. Mangin) Thom and Church ${ }^{3}$ & $\begin{array}{c}\text { Air, stone, wooden organ, pipes and } \\
\text { objects }\end{array}$ & {$[28,36,69,90,94]$} \\
\hline Aspergillus auricomus (Guég.) Saito & Air and graphic documents & [100] \\
\hline Aspergillus caespitosus Raper and Thom & Air, textiles and human remains & [83] \\
\hline Aspergillus calidoustus Varga, Houbraken and Samson & $\begin{array}{l}\text { Air, textiles and human remains } \\
\text { Air textiles, mummies, graphic }\end{array}$ & {$[28,83]$} \\
\hline Aspergillus candidus Link & $\begin{array}{l}\text { documents, maps, photographs and } \\
\text { human remains }\end{array}$ & {$[81,83,98-100]$} \\
\hline Aspergillus chevalieri (L. Mangin) Thom and Church ${ }^{4}$ & $\begin{array}{c}\text { Air, photographs, maps and graphic } \\
\text { documents }\end{array}$ & {$[99,100]$} \\
\hline Aspergillus clavatus Desm. & Air and textiles & {$[82,102]$} \\
\hline Aspergillus creber Jurjević, S.W. Peterson and B.W. Horn & Air & [28] \\
\hline Aspergillus cristatus Raper and Fennell ${ }^{5}$ & Funeral clothes, wooden organ and pipes & {$[85,94]$} \\
\hline Aspergillus fischeri Wehmer 6 & Air, textiles and human remains & [83] \\
\hline $\begin{array}{c}\text { Aspergillus flavipes (Bainier and R. Sartory) Thom } \\
\text { and Church }\end{array}$ & Air & {$[98,102]$} \\
\hline Aspergillus flavus Link $^{7}$ & $\begin{array}{c}\text { Air, stone, paintings, graphic documents, } \\
\text { photographs, maps, paintings, library } \\
\text { and museums }\end{array}$ & $\begin{array}{c}{[28,47,90,96,98-} \\
100,102,104]\end{array}$ \\
\hline Aspergillus fumigatus Fresen. & $\begin{array}{l}\text { Air, textiles, paper, parchment, funeral } \\
\text { clothes, libraries, museums and } \\
\text { human remains }\end{array}$ & {$[83,85,96,97]$} \\
\hline Aspergillus japonicus Saito & Air and graphic documents & [100] \\
\hline Aspergillus jensenii Jurjević, S.W. Peterson and B.W. Horn & Air & [28] \\
\hline Aspergillus melleus Yukawa & Air & [28] \\
\hline Aspergillus niger Tiegh & $\begin{array}{l}\text { Air, stone, paintings, libraries, museums, } \\
\text { graphic documents, photographs, maps, } \\
\text { drawings and lithographs }\end{array}$ & $\begin{array}{c}{[28,90,96,98-} \\
100,102,104,105]\end{array}$ \\
\hline Aspergillus ostianus Wehmer & Air and graphic documents & [100] \\
\hline Aspergillus P. Micheli ex Haller & Air & [47] \\
\hline Aspergillus penicillioides Speg. & Air and graphic documents & [100] \\
\hline Aspergillus protuberus Munt. -Cvetk. & Air & [28] \\
\hline Aspergillus pseudodeflectus Samson and Mouch. & Funeral clothes & [85] \\
\hline Aspergillus sydowii (Bainier and Sartory) Thom and Church & $\begin{array}{l}\text { Air, textiles, human remains, funeral } \\
\text { clothes, libraries, museums, wooden } \\
\text { organ and pipes }\end{array}$ & {$[28,83,85,94,96]$} \\
\hline Aspergillus tabacinus Nakaz., Y. Takeda, Simo and A. Watan. & Air & [28] \\
\hline Aspergillus tamarii Kita & $\begin{array}{l}\text { Air and materials present in libraries } \\
\text { and museums }\end{array}$ & [96] \\
\hline
\end{tabular}


Table 9. Cont.

\begin{tabular}{|c|c|c|}
\hline Current Species Name & Original Study Focus & References \\
\hline Aspergillus terreus Thom & $\begin{array}{l}\text { Air, textiles, wooden art objects, libraries, } \\
\text { museums and human remains }\end{array}$ & {$[36,69,83,96,102]$} \\
\hline $\begin{array}{c}\text { Aspergillus unguis (Émile-Weill and L. Gaudin) Thom } \\
\text { and Raper }\end{array}$ & Air and graphic documents & [100] \\
\hline Aspergillus unilateralis Thrower & Air and graphic documents & [100] \\
\hline Aspergillus ustus (Bainier) Thom and Church & Mummies and wooden art objects & {$[36,81]$} \\
\hline Aspergillus venenatus Jurjević, S.W. Peterson and B.W. Horn & Air, textiles and human remains & [83] \\
\hline Aspergillus versicolor (Vuill.) Tirab & $\begin{array}{l}\text { Air, wooden art objects, textiles, human } \\
\text { remains, albumen photographic } \\
\text { materials, drawings, lithographs, wooden } \\
\text { organ and pipes }\end{array}$ & $\begin{array}{c}{[28,36,83,93,94,102,} \\
105]\end{array}$ \\
\hline Aspergillus westerdijkiae Frisvad and Samson & Air, textiles and human remains & [83] \\
\hline Aureobasidium pullulans (de Bary and Löwenthal) G. Arnaud & Mummies & [81] \\
\hline Bjerkandera adusta (Willd.) P. Karst. & $\begin{array}{l}\text { Paper, parchment and albumen } \\
\text { photographic materials }\end{array}$ & {$[93,97]$} \\
\hline Chaetomium elatum Kunze & $\begin{array}{l}\text { Air, wooden art objects and albumen } \\
\text { photographic materials }\end{array}$ & {$[36,93,102]$} \\
\hline Chaetomium globosum Kunze & $\begin{array}{l}\text { Wooden art, air, paintings, libraries, } \\
\text { museums, drawings and lithographs }\end{array}$ & {$[36,69,96,103,105]$} \\
\hline Chaetomium thermophilum La Touche & Mummies & [81] \\
\hline $\begin{array}{c}\text { Cladosporium aggregatocicatricatum Bensch, Crous and } \\
\text { U. Braun }\end{array}$ & Wax seal & {$[84]$} \\
\hline $\begin{array}{l}\text { Cladosporium angustisporum Bensch, Summerell, Crous and } \\
\text { U. Braun }\end{array}$ & Drawings and lithographs & {$[105]$} \\
\hline Cladosporium cladosporioides (Fresen.) & $\begin{array}{l}\text { Air, wooden art objects, mummies, } \\
\text { libraries, museums, graphic documents, } \\
\text { photographs, maps, drawings. } \\
\text { lithographs, wooden organ and pipes }\end{array}$ & $\begin{array}{c}{[36,69,79,94,96,98-} \\
100,102,105]\end{array}$ \\
\hline Cladosporium herbarum (Pers.) Link $^{8}$ & Air and mummies & {$[81,102]$} \\
\hline Cladosporium Link & $\begin{array}{l}\text { Etruscan hypogeal tombs, air, textiles, } \\
\text { human remains, wooden art objects, } \\
\text { mummies, drawings and lithographs }\end{array}$ & {$[36,44,57,79,83,105]$} \\
\hline Cladosporium oxysporum Berk. and M.A. Curtis & $\begin{array}{l}\text { Air and materials present in libraries and } \\
\text { museums }\end{array}$ & {$[96,102]$} \\
\hline $\begin{array}{c}\text { Cladosporium pseudocladosporioides Bensch, Crous and U. } \\
\text { Braun }\end{array}$ & Mummies & {$[79]$} \\
\hline Cladosporium sphaerospermum Penz. & $\begin{array}{l}\text { Mummies, air and materials present in } \\
\text { libraries and museums }\end{array}$ & {$[79,82,92,96,105]$} \\
\hline Cladosporium tenuissimum Cooke & $\begin{array}{l}\text { Textiles, mummies, drawings and } \\
\text { lithographs }\end{array}$ & {$[79,82,105]$} \\
\hline Cladosporium uredinicola Speg. & Mummies & [79] \\
\hline Collariella bostrychodes (Zopf) X. Wei Wang and Samson ${ }^{9}$ & $\begin{array}{l}\text { Air and materials present in libraries and } \\
\text { museums }\end{array}$ & {$[96]$} \\
\hline Colletotrichum kahawae J.M. Waller and Bridge & Drawings and lithographs & [105] \\
\hline $\begin{array}{l}\text { Coniochaeta cipronana Coronado-Ruiz, Avendaño, } \\
\text { Escudero-Leyva, Conejo-Barboza, P. Chaverri and Chavarría }\end{array}$ & Drawings and lithographs & [105] \\
\hline $\begin{array}{c}\text { Coprinellus xanthothrix (Romagn.) Vilgalys, Hopple and Jacq. } \\
\text { Johnson }\end{array}$ & Air, textiles and human remains & [83] \\
\hline $\begin{array}{c}\text { Curoularia australiensis (Bugnic. ex M.B. Ellis) Manamgoda, } \\
\text { L. Cai and K.D. Hyde } 10\end{array}$ & Air and graphic documents & [100] \\
\hline Curvularia Boedijn & Air and stone & {$[47,90]$} \\
\hline Curvularia clavata B.L. Jain & Air & [102] \\
\hline Curvularia eragrostidis (Henn.) J.A. Mey. & Air and graphic documents & {$[98,100]$} \\
\hline Curvularia lunata (Wakker) Boedijn & $\begin{array}{l}\text { Air and materials present in libraries and } \\
\text { museums }\end{array}$ & {$[86,102]$} \\
\hline Curvularia pallescens Boedijn & Air and graphic documents & {$[98,100,102]$} \\
\hline
\end{tabular}


Table 9. Cont

\begin{tabular}{|c|c|c|}
\hline Current Species Name & Original Study Focus & References \\
\hline Cyphellophora G.A. de Vries & Etruscan hypogeal tombs & [44] \\
\hline Cyphellophora olivacea (W. Gams) Réblová \& Unter. & Etruscan hypogeal tombs & [44] \\
\hline Dichotomopilus indicus (Corda) X. Wei Wang and Samson ${ }^{11}$ & $\begin{array}{c}\text { Air and materials present in libraries and } \\
\text { museums }\end{array}$ & [96] \\
\hline Dipodascus Lagerh. ${ }^{12}$ & Air & [47] \\
\hline Epicoccum Link & Air and graphic documents & [100] \\
\hline Epicoccum nigrum Link & Mummies, wooden organ and pipes & {$[79,94]$} \\
\hline Fomes fomentarius (L.) Fr. & Textiles & [82] \\
\hline Fulvia fulva (Cooke) Cif. ${ }^{13}$ & Air & [102] \\
\hline Fusarium Link & Air, stone and graphic documents & {$[47,90,100,102]$} \\
\hline Fusarium oxysporum Schltdl. & Air and wooden materials & {$[70,98]$} \\
\hline $\begin{array}{c}\text { Fusarium proliferatum (Matsush.) Nirenberg ex Gerlach and } \\
\text { Nirenberg }\end{array}$ & Air & {$[98]$} \\
\hline Hormodendrum pyri W.H. English & Wax seal & [84] \\
\hline Hypochnicium J. Erikss. & Wooden materials & [61] \\
\hline Meyerozyma guilliermondii (Wick.) Kurtzman and M. Suzuki & Air & {$[98]$} \\
\hline Microascus brevicaulis S.P. Abbott 15 & Air, textiles and human remains & [83] \\
\hline Mortierella Coem. & Wooden materials & [61] \\
\hline Mucor circinelloides Tiegh. & Mummies & [81] \\
\hline Mucor P. Micheli ex L. & Air & [102] \\
\hline Mucor plumbeus Bonord. & Albumen photographic materials & [93] \\
\hline Mucor racemosus Bull. & Air, paper and parchment & {$[97,98]$} \\
\hline Mucor spinosus Schrank & Paper and parchment & [97] \\
\hline "Neocosmospora" solani (Mart.) L. Lombard and Crous 16 & Wooden materials & [70] \\
\hline Neurospora crassa Shear and B.O. Dodge & Air & {$[98,102]$} \\
\hline Neurospora sitophila Shear and B.O. Dodge ${ }^{17}$ & $\begin{array}{l}\text { Air and materials present in libraries and } \\
\text { museums }\end{array}$ & {$[96,98]$} \\
\hline Nigrospora oryzae (Berk. and Broome) Petch & Air, textiles and human remains & [83] \\
\hline Nigrospora oryzae (Berk. and Broome) Petch & $\begin{array}{l}\text { Air and materials present in libraries and } \\
\text { museums }\end{array}$ & {$[96,98]$} \\
\hline Paecilomyces maximus C. Ram ${ }^{18}$ & Wooden organ and pipes & [94] \\
\hline Paecilomyces variotii Bainier & Air & [98] \\
\hline Penicillium aurantiogriseum Dierckx & Air & [102] \\
\hline Penicillium brevicompactum Dierckx & Air & [28] \\
\hline Penicillium camemberti Thom & Paper and parchment & [97] \\
\hline Penicillium canescens Sopp & Air and graphic documents & {$[28,100]$} \\
\hline Penicillium carneum (Frisvad) Frisvad & Air & [28] \\
\hline Penicillium chrysogenum Thom & $\begin{array}{l}\text { Wooden objects, air, textiles, mummies, } \\
\text { human remains, drawings, maps, } \\
\text { photographs, lithographs, graphic } \\
\text { documents and materials present in } \\
\text { libraries and museums }\end{array}$ & $\begin{array}{c}{[28,36,69,79,82,83,96-} \\
100,102,105]\end{array}$ \\
\hline Penicillium citreonigrum Dierckx & Air & [28] \\
\hline Penicillium citrinum Thom & $\begin{array}{l}\text { Air, paintings, graphic documents, } \\
\text { photographs, maps and materials present } \\
\text { in libraries and museums }\end{array}$ & {$[28,96,99,100,102,104]$} \\
\hline Penicillium commune Thom & $\begin{array}{l}\text { Air, textiles, paper, parchment, funeral } \\
\text { clothes and human remains }\end{array}$ & {$[83,85,97,102]$} \\
\hline Penicillium corylophilum Dierckx & $\begin{array}{l}\text { Textiles, air and materials present in } \\
\text { libraries and museums }\end{array}$ & {$[82,96]$} \\
\hline Penicillium crocicola T. Yamam. & Funeral clothes & [85] \\
\hline Penicillium crustosum Thom & $\begin{array}{l}\text { Air, textiles, funeral clothes, human } \\
\text { remains and wooden organ and pipes }\end{array}$ & {$[83,85,94]$} \\
\hline Penicillium decumbens Thom 19 & Air, paper and parchment & {$[28,97,102]$} \\
\hline Penicillium digitatum (Pers.) Sacc. & Air, textiles and human remains & {$[28,83,102]$} \\
\hline
\end{tabular}


Table 9. Cont.

\begin{tabular}{|c|c|c|}
\hline Current Species Name & Original Study Focus & References \\
\hline Penicillium expansum Link & $\begin{array}{l}\text { Air, wooden art objects and } \\
\text { funeral clothes }\end{array}$ & {$[28,36,69,85]$} \\
\hline Penicillium glabrum (Wehmer) Westling & $\begin{array}{l}\text { Wooden objects, air and materials present } \\
\text { in libraries and museums }\end{array}$ & {$[28,69,96]$} \\
\hline Penicillium griseofulvum Dierckx & $\begin{array}{l}\text { Air, textiles, graphic documents and } \\
\text { human remains }\end{array}$ & {$[83,100,102]$} \\
\hline Penicillium herquei Bainier and Sartory & Wooden objects and air & {$[36,69]$} \\
\hline Penicillium janczewskii K.W. Zaleski & $\begin{array}{c}\text { Air, photographs, maps and graphic } \\
\text { documents }\end{array}$ & {$[99,100]$} \\
\hline Penicillium Link & $\begin{array}{l}\text { Air, mummies, wooden art objects, } \\
\text { albumen photographic materials, funeral } \\
\text { accessories and paintings }\end{array}$ & $\begin{array}{c}{[36,47,80,81,93,98-} \\
100,103]\end{array}$ \\
\hline Penicillium raistrickii G. Sm. & Mummies & [79] \\
\hline Penicillium roseopurpureum Dierckx & Mummies and funeral clothes & {$[79,85]$} \\
\hline Penicillium rubens Biourge & Painting & [103] \\
\hline Penicillium sacculum E. Dale & Wooden objects and air & {$[36,69]$} \\
\hline Penicillium sanguifluum (Sopp) Biourge & Air & [28] \\
\hline Penicillium simplicissimum (Oudem.) Thom & $\begin{array}{l}\text { Air, photographs, maps, graphic } \\
\text { materials and materials present in } \\
\text { libraries and museums }\end{array}$ & {$[96,99,100]$} \\
\hline Penicillium solitum Westling & Air & [28] \\
\hline Penicillium thomii Maire & Albumen photographic materials & [93] \\
\hline Penicillium ulaiense H.M. Hsieh, H.J. Su and Tzean & Air & [28] \\
\hline $\begin{array}{l}\text { Penicillium viridicatum } \\
\text { Westling }\end{array}$ & Paper and parchment & [97] \\
\hline Penicillium waksmanii K.W. Zaleski & Air and graphic documents & [100] \\
\hline Penicillium westlingii K.W. Zaleski & Drawings and lithographs & [105] \\
\hline $\begin{array}{c}\text { Periconia epigraphicola Coronado-Ruiz, R. E. Escudero-Leyva, } \\
\text { G. Conejo-Barboza, P. Chaverri and M. Chavarría }\end{array}$ & Drawings and lithographs & [105] \\
\hline Pestalotia De Not. & Air and graphic documents & [100] \\
\hline Phlebia Fr. & Albumen photographic materials & [93] \\
\hline Phoma herbarum Westend. & Paper and parchment & [97] \\
\hline Pleosporales Luttr. ex M.E. Barr & Albumen photographic materials & [93] \\
\hline Pleurotus ostreatus (Jacq.) P. Kumm. ${ }^{20}$ & Albumen photographic materials & [93] \\
\hline $\begin{array}{c}\text { Pseudallescheria fimeti (Arx, Mukerji and N. Singh) McGinnis, } \\
\text { A.A. Padhye and Ajello }\end{array}$ & Painting & [103] \\
\hline Pseudallescheria Negr. and I. Fisch. & Painting & [103] \\
\hline $\begin{array}{c}\text { Pseudogymnoascus pannorum (Link) Minnis and D.L. } \\
\text { Lindner } 21\end{array}$ & Wooden objects and air & {$[36,69]$} \\
\hline $\begin{array}{c}\text { Pseudoscopulariopsis hibernica (A. Mangan) Sand. -Den., Gené } \\
\text { and Cano } 22\end{array}$ & Air, textiles and human remains & [83] \\
\hline Rhizopus arrhizus A. Fisch. ${ }^{23}$ & Air, textiles and human remains & [83] \\
\hline Rhizopus microsporus Tiegh. & Air, textiles and human remains & [83] \\
\hline Sarocladium W. Gams and D. Hawksw. ${ }^{24}$ & Air and graphic documents & {$[98,100]$} \\
\hline Sporobolomyces roseus Kluyver and C.B. Niel 25 & Funeral accessories & [80] \\
\hline Stachybotrys chartarum (Ehrenb.) S. Hughes & Mummies & [81] \\
\hline Stemphylium vesicarium (Wallr.) E.G. Simmons & Mummies & [79] \\
\hline $\begin{array}{c}\text { Syncephalastrum racemosum } \\
\text { Cohn ex J. Schröt. }\end{array}$ & $\begin{array}{l}\text { Air and materials present in libraries and } \\
\text { museums }\end{array}$ & [96] \\
\hline $\begin{array}{c}\text { Talaromyces amestolkiae N. Yilmaz, Houbraken, Frisvad } \\
\text { and Samson }\end{array}$ & Air & [28] \\
\hline $\begin{array}{c}\text { Talaromyces purpureogenus Samson, N. Yilmaz, Houbraken, } \\
\text { Spierenb., Seifert, Peterson, Varga and Frisvad }\end{array}$ & Stone & {$[90]$} \\
\hline $\begin{array}{c}\text { Talaromyces rugulosus (Thom) Samson, N. Yilmaz, Frisvad } \\
\text { and Seifert }\end{array}$ & Wooden organ and pipes & [94] \\
\hline $\begin{array}{c}\text { Talaromyces sayulitensis Visagie, N. Yilmaz, Seifert } \\
\text { and Samson }\end{array}$ & Air & [28] \\
\hline
\end{tabular}


Table 9. Cont.

\begin{tabular}{|c|c|c|}
\hline Current Species Name & Original Study Focus & References \\
\hline $\begin{array}{c}\text { Talaromyces verruculosus (Peyronel) Samson, N. Yilmaz, } \\
\text { Frisvad and Seifert }\end{array}$ & Air & [28] \\
\hline Trichoderma harzianum Rifai & Air, textiles and human remains & [83] \\
\hline Trichoderma longibrachiatum Rifai & Drawings and lithographs & [105] \\
\hline Trichoderma viride Pers. & Wooden art objects and air & {$[36,69,98]$} \\
\hline Trichothecium roseum (Pers.) Link & Albumen photographic materials & [93] \\
\hline
\end{tabular}

Previously identified as: ${ }^{1}$ Ulocladium chartarum; ${ }^{2}$ Alternaria consortiale; ${ }^{3}$ Eurotium amsteldomi ${ }^{4}$ Eurotium chevalieri; ${ }^{5}$ Eurotium cristatum; ${ }^{6}$ Neosartorya fischeri; ${ }^{7}$ Aspergillus oryzae; ${ }^{8}$ Cladosporium herbarium; ${ }^{9}$ Chaetomium bostrychodes; ${ }^{10}$ Bipolaris australiensis; ${ }^{11}$ Chaetomium indicum;

${ }^{12}$ Geotrichum sp.; ${ }^{13}$ Cladosporium fulvum; ${ }^{14}$ Candida guilliermondii; ${ }^{15}$ Scopulariopsis brevicaulis; ${ }^{16}$ Fusarium solani; ${ }^{17}$ Chrysonilia sitophila;

${ }^{18}$ Paecilomyces formosus; ${ }^{19}$ Penicillium funiculosum; ${ }^{20}$ Pleurotus pulmonarius; ${ }^{21}$ Chrysosporium pannorum; ${ }^{22}$ Scopulariopsis hibernica; ${ }^{23}$ Rhizopus oryzae; ${ }^{24}$ Cephalosporium sp.; ${ }^{25}$ Sporidiobolus metaroseus.

\section{Conclusions}

As pointed and reviewed by Pyzik and colleagues [107] the application of highthroughput Next-Generation sequencing technologies has highlighted that cultural heritage materials are inhabited by various unknown microorganisms still pending taxonomic description and their biodeteriorative profiling. The material biodeterioration is known to sometimes be caused by a predominant or specific microbial group, while more often the complex biodeterioration processes are a result of the synergistic action of a group of organisms resulting from various colonization events influenced by the impacts of multiple external factors throughout a time frame [77]. Cultivation methodologies often face limitations in what regards the ability for distinct organisms to be effectively cultivated and their original biodeteriorative characteristics replicated under laboratory conditions [108]. Understandably the application of more modern molecular techniques in cultural heritage biodeterioration studies has been increasingly being used and updated for the last two decades $[27,108]$. Although with their own set of limitations, culture-dependent methodologies still offer three main advantages: (1) The isolation of microbes for further differential analysis; (2) the possibility to isolate, characterize and describe previously unknown taxa; and (3) the development and improvement of biological and genetic databases. These aspects are especially important when considering that even the biodeteriorative role (but also their taxonomic classification) of long known species might also need to be constantly revised, updated and reevaluated $[107,109]$. For instance, the inclusion of the Fusarium solani Species Complex in the genus Neocosmospora was recently reevaluated and continues to be the focus of additional studies [110].

Fungi are constantly regarded as one if not the most important microorganism groups causing cultural heritage materials biodeterioration $[2,3,30]$. This review highlighted that, so far, isolates from more than two-hundred fungal species have been showed to exhibit biodeteriorative abilities when studied by specific plate essays. Based on the available studies performed so far, it is possible to verify that Aspergillus and Penicillium species dominate the biodeteriorative abilities usually screened in biodeterioration contexts. With this in mind, it should be reinforced that the detection of these species in various cultural heritage materials can, under specific conditions, result in severe biodeterioration of the substrate. Nonetheless, a careful analysis of these checklists, as well as, the biodeteriorative screening of obtained isolates, wherever possible, is strongly advised. Not all isolates might display deteriorative action or display similar degradative rates and thus a proper and specific analysis in each case and/or the implementation of additional tests (e.g., molecular identification of genes involved in biodeterioration (see for example [111])) is also recommended. In conjunction with molecular approaches not relying in cultivation, they can provide a holistic evaluation of a specific biodeterioration phenomena. As pointed by Sterflinger and colleagues [112], understanding deterioration mechanisms and the main microbial perpetrators is still one of the major challenges in historic and cultural materials biodeterioration research. As such, the information summarized in this work provides a 
contribution that can help microbiologists, restorers, conservators and curators in their attempt to preserve cultural heritage materials for future generations.

Author Contributions: Conceptualization, J.T. writing—original draft preparation, J.T.; writingreview and editing, J.T. and A.P.; supervision, A.P.; funding acquisition, A.P. All authors have read and agreed to the published version of the manuscript.

Funding: This work was financed by IPN-Financiamento Base FITEC approved under the National Call with reference no. 01/FITEC/2018 to obtain multi-year base financing under the INTERFACE Program and by FEDER- Fundo Europeu de Desenvolvimento Regional funds through the COMPETE 2020-Operational Programme for Competitiveness and Internationalisation (POCI), and by Portuguese funds through FCT- Fundação para a Ciência e a Tecnologia in the framework of the project POCI-01-0145-FEDER-PTDC/EPH-PAT/3345/2014. This work was carried out at the R\&D Unit Centre for Functional Ecology-Science for People and the Planet (CFE), with reference UIDB/04004/2020, financed by FCT/MCTES through national funds (PIDDAC). João Trovão was supported by $\mathrm{POCH}$-Programa Operacional Capital Humano (co-funding by the European Social Fund and national funding by MCTES), through a "FCT- Fundação para a Ciência e Tecnologia" PhD research grant (SFRH/BD/132523/2017).

Institutional Review Board Statement: Not applicable.

Informed Consent Statement: Not applicable.

Data Availability Statement: Not applicable.

Conflicts of Interest: The authors declare no conflict of interest.

\section{References}

1. Deacon, J.W. Fungal Biology, 4th ed.; Blackwell Pub.: Malden, MA, USA, 2006.

2. Sterflinger, K. Fungi: Their Role in Deterioration of Cultural Heritage. Fungal Biol. Rev. 2010, 24, 47-55. [CrossRef]

3. Sterflinger, K.; Piñar, G. Microbial Deterioration of Cultural Heritage and Works of Art-Tilting at Windmills? Appl. Microbiol. Biotechnol. 2013, 97, 9637-9646. [CrossRef]

4. Dakal, T.C.; Cameotra, S.S. Microbially Induced Deterioration of Architectural Heritages: Routes and Mechanisms Involved. Environ. Sci. Eur. 2012, 24, 36. [CrossRef]

5. Gadd, G.M. Geomicrobiology of the Built Environment. Nat. Microbiol. 2017, 2, 1-9. [CrossRef]

6. Gadd, G.M. Fungi, Rocks, and Minerals. Elements 2017, 13, 171-176. [CrossRef]

7. Cappitelli, F.; Sorlini, C. From Papyrus to Compact Disc: The Microbial Deterioration of Documentary Heritage. Crit. Rev. Microbiol. 2005, 31, 1-10. [CrossRef]

8. Sterflinger, K.; Pinzari, F. The Revenge of Time: Fungal Deterioration of Cultural Heritage with Particular Reference to Books, Paper and Parchment. Environ. Microbiol. 2012, 14, 559-566. [CrossRef]

9. Paiva de Carvalho, H.; Mesquita, N.; Trovão, J.; Fernández Rodríguez, S.; Pinheiro, A.C.; Gomes, V.; Alcoforado, A.; Gil, F.; Portugal, A. Fungal Contamination of Paintings and Wooden Sculptures inside the Storage Room of a Museum: Are Current Norms and Reference Values Adequate? J. Cult. Herit. 2018, 34, 268-276. [CrossRef]

10. Poyatos, F.; Morales, F.; Nicholson, A.W.; Giordano, A. Physiology of Biodeterioration on Canvas Paintings. J. Cell Physiol. 2018, 233, 2741-2751. [CrossRef] [PubMed]

11. Kosel, J.; Ropret, P. Overview of Fungal Isolates on Heritage Collections of Photographic Materials and Their Biological Potency. J. Cult. Herit. 2021, 48, 277-291. [CrossRef]

12. Coutinho, M.L.; Miller, A.Z.; Macedo, M.F. Biological Colonization and Biodeterioration of Architectural Ceramic Materials: An Overview. J. Cult. Herit. 2015, 16, 759-777. [CrossRef]

13. Sclocchi, M.C.; Kraková, L.; Pinzari, F.; Colaizzi, P.; Bicchieri, M.; Šaková, N.; Pangallo, D. Microbial Life and Death in a Foxing Stain: A Suggested Mechanism of Photographic Prints Defacement. Microb. Ecol. 2017, 73, 815-826. [CrossRef]

14. Piñar, G.; Piombino-Mascali, D.; Maixner, F.; Zink, A.; Sterflinger, K. Microbial Survey of the Mummies from the Capuchin Catacombs of Palermo, Italy: Biodeterioration Risk and Contamination of the Indoor Air. FEMS Microbiol. Ecol. 2013, 86, 341-356. [CrossRef] [PubMed]

15. Ruga, L.; Orlandi, F.; Romano, B.; Fornaciari, M. The Assessment of Fungal Bioaerosols in the Crypt of St. Peter in Perugia (Italy). Int. Biodeterior. Biodegrad. 2015, 98, 121-130. [CrossRef]

16. Rodrigues, A.; Gutierrez-Patricio, S.; Miller, A.Z.; Saiz-Jimenez, C.; Wiley, R.; Nunes, D.; Vilarigues, M.; Macedo, M.F. Fungal Biodeterioration of Stained-Glass Windows. Int. Biodeterior. Biodegrad. 2014, 90, 152-160. [CrossRef]

17. Beata, G. The Use of -Omics Tools for Assessing Biodeterioration of Cultural Heritage: A Review. J. Cult. Herit. 2020 , 45, 351-361. [CrossRef] 
18. Amann, R.I.; Ludwig, W.; Schleifer, K.H. Phylogenetic Identification and in Situ Detection of Individual Microbial Cells without Cultivation. Microbiol. Rev. 1995, 59, 143-169. [CrossRef]

19. Dakal, T.C.; Arora, P.K. Evaluation of Potential of Molecular and Physical Techniques in Studying Biodeterioration. Rev. Environ. Sci. Biotechnol. 2012, 11, 71-104. [CrossRef]

20. González Grau, J.M.; Sáiz-Jiménez, C. Unknown Microbial Communities on Rock Art Paintings. Consequences for Conservation and Future Perspectives. Coalition 2005, 10, 4-7.

21. Gonzalez, J.M.; Saiz-Jimenez, C. Microbial Diversity in Biodeteriorated Monuments as Studied by Denaturing Gradient Gel Electrophoresis. J. Sep. Sci. 2004, 27, 174-180. [CrossRef]

22. González, J.M.; Sáiz-Jiménez, C. Application of Molecular Nucleic Acid-Based Techniques for the Study of Microbial Communities in Monuments and Artworks. Int. Microbiol. 2005, 8, 189-194. [PubMed]

23. Laiz, L.; Piñar, G.; Lubitz, W.; Sáiz-Jiménez, C. The Colonisation of Building Materials by Microorganisms as Revealed by Culturing and Molecular Methods. Mol. Biol. Cult. Herit. 2003. [CrossRef]

24. Mihajlovski, A.; Seyer, D.; Benamara, H.; Bousta, F.; Di Martino, P. An Overview of Techniques for the Characterization and Quantification of Microbial Colonization on Stone Monuments. Ann. Microbiol. 2015, 65, 1243-1255. [CrossRef]

25. Otlewska, A.; Adamiak, J.; Gutarowska, B. Application of Molecular Techniques for the Assessment of Microorganism Diversity on Cultural Heritage Objects. Acta Biochim. Pol. 2014, 61. [CrossRef]

26. Sanmartín, P.; DeAraujo, A.; Vasanthakumar, A. Melding the Old with the New: Trends in Methods Used to Identify, Monitor, and Control Microorganisms on Cultural Heritage Materials. Microb. Ecol. 2018, 76, 64-80. [CrossRef] [PubMed]

27. Ward, D.M.; Weller, R.; Bateson, M.M. 16S RRNA Sequences Reveal Numerous Uncultured Microorganisms in a Natural Community. Nature 1990, 345, 63-65. [CrossRef]

28. Savković, Ž.; Stupar, M.; Unković, N.; Ivanović, Ž.; Blagojević, J.; Vukojević, J.; Ljaljević Grbić, M. In Vitro Biodegradation Potential of Airborne Aspergilli and Penicillia. Sci. Nat. 2019, 106, 8. [CrossRef] [PubMed]

29. Warscheid, T.; Braams, J. Biodeterioration of Stone: A Review. Int. Biodeterior. Biodegrad. 2000, 46, 343-368. [CrossRef]

30. Sterflinger, K. Fungi as Geologic Agents. Geomicrobiol. J. 2000, 17, 97-124. [CrossRef]

31. Scheerer, S.; Ortega-Morales, O.; Gaylarde, C. Chapter 5 Microbial Deterioration of Stone Monuments-An Updated Overview. Adv. Appl. Microbiol. 2009, 66, 97-139. [CrossRef]

32. Liu, X.; Koestler, R.J.; Warscheid, T.; Katayama, Y.; Gu, J.-D. Microbial Deterioration and Sustainable Conservation of Stone Monuments and Buildings. Nat. Sustain. 2020, 3, 991-1004. [CrossRef]

33. Albertano, P.; Urzì, C. Structural Interactions among Epilithic Cyanobacteria and Heterotrophic Microorganisms in Roman Hypogea. Microb. Ecol. 1999, 38, 244-252. [CrossRef] [PubMed]

34. Burford, E.P.; Kierans, M.; Gadd, G.M. Geomycology: Fungi in Mineral Substrata. Mycologist 2003, 17, 98-107. [CrossRef]

35. Ortega-Morales, B.O.; Narváez-Zapata, J.; Reyes-Estebanez, M.; Quintana, P.; la Rosa-García, D.; Bullen, H.; Gómez-Cornelio, S.; Chan-Bacab, M.J. Bioweathering Potential of Cultivable Fungi Associated with Semi-Arid Surface Microhabitats of Mayan Buildings. Front. Microbiol. 2016, 7, 201. [CrossRef] [PubMed]

36. Pangallo, D.P.; Chovanová, K.C.; Šimonovičová, A.Š.; Ferianc, P.F. Investigation of Microbial Community Isolated from Indoor Artworks and Air Environment: Identification, Biodegradative Abilities, and DNA Typing. Can. J. Microbiol. 2009, 55, $277-287$. [CrossRef] [PubMed]

37. Kiyuna, T.; An, K.-D.; Kigawa, R.; Sano, C.; Miura, S.; Sugiyama, J. Bristle-like Fungal Colonizers on the Stone Walls of the Kitora and Takamatsuzuka Tumuli Are Identified as Kendrickiella Phycomyces. Mycoscience 2012, 53, 446-459. [CrossRef]

38. Unković, N.; Dimkić, I.; Stupar, M.; Stanković, S.; Vukojević, J.; Grbić, M.L. Biodegradative Potential of Fungal Isolates from Sacral Ambient: In Vitro Study as Risk Assessment Implication for the Conservation of Wall Paintings. PLoS ONE 2018, 13, e0190922. [CrossRef]

39. Ponizovskaya, V.B.; Rebrikova, N.L.; Kachalkin, A.V.; Antropova, A.B.; Bilanenko, E.N.; Mokeeva, V.L. Micromycetes as Colonizers of Mineral Building Materials in Historic Monuments and Museums. Fungal Biol. 2019, 123, 290-306. [CrossRef]

40. Gámez-Espinosa, E.; Bellotti, N.; Deyá, C.; Cabello, M. Mycological Studies as a Tool to Improve the Control of Building Materials Biodeterioration. J. Build. Eng. 2020, 32, 101738. [CrossRef]

41. Ma, W.; Wu, F.; Tian, T.; He, D.; Zhang, Q.; Gu, J.-D.; Duan, Y.; Ma, D.; Wang, W.; Feng, H. Fungal Diversity and Its Contribution to the Biodeterioration of Mural Paintings in Two 1700-Year-Old Tombs of China. Int. Biodeterior. Biodegrad. 2020, $152,104972$. [CrossRef]

42. Trovão, J.; Tiago, I.; Catarino, L.; Gil, F.; Portugal, A. In Vitro Analyses of Fungi and Dolomitic Limestone Interactions: Bioreceptivity and Biodeterioration Assessment. Int. Biodeterior. Biodegrad. 2020, 155, 105107. [CrossRef]

43. Trovão, J.; Gil, F.; Catarino, L.; Soares, F.; Tiago, I.; Portugal, A. Analysis of Fungal Deterioration Phenomena in the First Portuguese King Tomb Using a Multi-Analytical Approach. Int. Biodeterior. Biodegrad. 2020, 149, 104933. [CrossRef]

44. Isola, D.; Zucconi, L.; Cecchini, A.; Caneva, G. Dark-Pigmented Biodeteriogenic Fungi in Etruscan Hypogeal Tombs: New Data on Their Culture-Dependent Diversity, Favouring Conditions, and Resistance to Biocidal Treatments. Fungal Biol. 2021. [CrossRef]

45. De Ley, J.; Swings, J.; Gosselé, F. Genus I. Acetobacter Beijerinck 1898, 215 ${ }^{\mathrm{AL}}$. In Bergey's Manual of Systematic Bacteriology; Williams and Wilkins: Baltimore, MD, USA, 1984; Volume 1, pp. 268-274.

46. Samson, R.A.; Houbraken, J.; Thrane, U.; Frisvad, J.C.; Andersen, B. Food and Indoor Fungi, 2nd ed.; CBS-KNAW Fungal Biodiversity Centre: Utrecht, The Netherlands, 2010. 
47. Borrego, S.; Guiamet, P.; Gómez de Saravia, S.; Batistini, P.; Garcia, M.; Lavin, P.; Perdomo, I. The Quality of Air at Archives and the Biodeterioration of Photographs. Int. Biodeterior. Biodegrad. 2010, 64, 139-145. [CrossRef]

48. Gutarowska, B. Metabolic Activity of Moulds as a Factor of Building Materials Biodegradation. Pol. J. Microbiol. 2010, 59, 119-124. [CrossRef]

49. Ahmad, A.; Rautaray, D.; Sastry, M. Biogenic Calcium Carbonate: Calcite Crystals of Variable Morphology by the Reaction of Aqueous $\mathrm{Ca}^{2+}$ Ions with Fungi. Adv. Funct. Mater. 2004, 14, 1075-1080. [CrossRef]

50. Burford, E.P.; Hillier, S.; Gadd, G.M. Biomineralization of Fungal Hyphae with Calcite (CaCO3) and Calcium Oxalate Mono- and Dihydrate in Carboniferous Limestone Microcosms. Geomicrobiol. J. 2006, 23, 599-611. [CrossRef]

51. Gadd, G.M.; Bahri-Esfahani, J.; Li, Q.; Rhee, Y.J.; Wei, Z.; Fomina, M.; Liang, X. Oxalate Production by Fungi: Significance in Geomycology, Biodeterioration and Bioremediation. Fungal Biol. Rev. 2014, 28, 36-55. [CrossRef]

52. Del Monte, M.; Sabbioni, C.; Zappia, G. The Origin of Calcium Oxalates on Historical Buildings, Monuments and Natural Outcrops. Sci. Total Environ. 1987, 67, 17-39. [CrossRef]

53. Li, T.; Hu, Y.; Zhang, B.; Yang, X. Role of Fungi in the Formation of Patinas on Feilaifeng Limestone, China. Microb. Ecol. 2018, 76, 352-361. [CrossRef]

54. Savković, Ž.; Unković, N.; Stupar, M.; Franković, M.; Jovanović, M.; Erić, S.; Šarić, K.; Stanković, S.; Dimkić, I.; Vukojević, J.; et al. Diversity and Biodeteriorative Potential of Fungal Dwellers on Ancient Stone Stela. Int. Biodeterior. Biodegrad. 2016, 115, $212-223$. [CrossRef]

55. Li, T.; Hu, Y.; Zhang, B. Biomineralization Induced by Colletotrichum acutatum: A Potential Strategy for Cultural Relic Bioprotection. Front. Microbiol. 2018, 9, 1884. [CrossRef]

56. Unković, N.; Erić, S.; Šarić, K.; Stupar, M.; Savković, Ž.; Stanković, S.; Stanojević, O.; Dimkić, I.; Vukojević, J.; Ljaljević Grbić, M. Biogenesis of Secondary Mycogenic Minerals Related to Wall Paintings Deterioration Process. Micron 2017, 100, 1-9. [CrossRef]

57. Boquet, E.; Boronat, A.; Ramos-Cormenzana, A. Production of Calcite (Calcium Carbonate) Crystals by Soil Bacteria Is a General Phenomenon. Nature 1973, 246, 527-529. [CrossRef]

58. Guggiari, M.; Bloque, R.; Aragno, M.; Verrecchia, E.; Job, D.; Junier, P. Experimental Calcium-Oxalate Crystal Production and Dissolution by Selected Wood-Rot Fungi. Int. Biodeterior. Biodegrad. 2011, 65, 803-809. [CrossRef]

59. Pasquale, V.; Fiore, S.; Hlayem, D.; Lettino, A.; Huertas, F.J.; Chianese, E.; Dumontet, S. Biomineralization of Carbonates Induced by the Fungi Paecilomyces inflatus and Plectosphaerella cucumerina. Int. Biodeterior. Biodegrad. 2019, 140, 57-66. [CrossRef]

60. Gadd, G.M. Geomycology: Biogeochemical Transformations of Rocks, Minerals, Metals and Radionuclides by Fungi, Bioweathering and Bioremediation. Mycol. Res. 2007, 111, 3-49. [CrossRef] [PubMed]

61. Liu, Z.; Wang, Y.; Pan, X.; Ge, Q.; Ma, Q.; Li, Q.; Fu, T.; Hu, C.; Zhu, X.; Pan, J. Identification of Fungal Communities Associated with the Biodeterioration of Waterlogged Archeological Wood in a Han Dynasty Tomb in China. Front. Microbiol. 2017, 8. [CrossRef]

62. Gutarowska, B.; Celikkol-Aydin, S.; Bonifay, V.; Otlewska, A.; Aydin, E.; Oldham, A.L.; Brauer, J.I.; Duncan, K.E.; Adamiak, J.; Sunner, J.A.; et al. Metabolomic and High-Throughput Sequencing Analysis-Modern Approach for the Assessment of Biodeterioration of Materials from Historic Buildings. Front. Microbiol. 2015, 6, 979. [CrossRef] [PubMed]

63. Ortiz, R.; Navarrete, H.; Navarrete, J.; Párraga, M.; Carrasco, I.; de la Vega, E.; Ortiz, M.; Herrera, P.; Blanchette, R.A. Deterioration, Decay and Identification of Fungi Isolated from Wooden Structures at the Humberstone and Santa Laura Saltpeter Works: A World Heritage Site in Chile. Int. Biodeterior. Biodegrad. 2014, 86, 309-316. [CrossRef]

64. Piñar, G.; Dalnodar, D.; Voitl, C.; Reschreiter, H.; Sterflinger, K. Biodeterioration Risk Threatens the 3100 Year Old Staircase of Hallstatt (Austria): Possible Involvement of Halophilic Microorganisms. PLoS ONE 2016, 11, e0148279. [CrossRef] [PubMed]

65. Pournou, A. Biodeterioration of Wooden Cultural Heritage: Organisms and Decay Mechanisms in Aquatic and Terrestrial Ecosystems; Springer International Publishing: Cham, Switzerland, 2020; ISBN 978-3-030-46503-2.

66. Alfieri, P.V.; García, R.A.; Rosato, V.G.; Correa, M.V. Biodeterioration and Biodegradation of Wooden Heritage: Role of Fungal Succession. Int. J. Conserv. Sci. 2016, 7, 607-614.

67. Blanchette, R.A. A Review of Microbial Deterioration Found in Archaeological Wood from Different Environments. Int. Biodeterior. Biodegrad. 2000, 46, 189-204. [CrossRef]

68. Goodell, B.; Winandy, J.E.; Morrell, J.J. Fungal Degradation of Wood: Emerging Data, New Insights and Changing Perceptions. Coatings 2020, 10, 1210. [CrossRef]

69. Pangallo, D.; Šimonovičová, A.; Chovanová, K.; Ferianc, P. Wooden Art Objects and the Museum Environment: Identification and Biodegradative Characteristics of Isolated Microflora. Lett. Appl. Microbiol. 2007, 45, 87-94. [CrossRef]

70. Liu, Z.; Fu, T.; Hu, C.; Shen, D.; Macchioni, N.; Sozzi, L.; Chen, Y.; Liu, J.; Tian, X.; Ge, Q.; et al. Microbial Community Analysis and Biodeterioration of Waterlogged Archaeological Wood from the Nanhai No. 1 Shipwreck during Storage. Sci. Rep. 2018, 8, 7170. [CrossRef] [PubMed]

71. Archibald, F.S. A New Assay for Lignin-Type Peroxidases Employing the Dye Azure B. Appl. Environ. Microbiol. 1992, 58, 3110-3116. [CrossRef]

72. Manji, S.; Ishihara, A. Screening of Tetrachlorodibenzo-p-Dioxin-Degrading Fungi Capable of Producing Extracellular Peroxidases under Various Conditions. Appl. Microbiol. Biotechnol. 2004, 63, 438-444. [CrossRef]

73. Falade, A.O.; Eyisi, O.A.L.; Mabinya, L.V.; Nwodo, U.U.; Okoh, A.I. Peroxidase Production and Ligninolytic Potentials of Fresh Water Bacteria Raoultella Ornithinolytica and Ensifer Adhaerens. Biotechnol. Rep. 2017, 16, 12-17. [CrossRef] 
74. Kiiskinen, L.-L.; Rättö, M.; Kruus, K. Screening for Novel Laccase-Producing Microbes. J. Appl. Microbiol. 2004, 97, 640-646. [CrossRef]

75. Szostak-Kotowa, J. Biodeterioration of Textiles. Int. Biodeterior. Biodegrad. 2004, 53, 165-170. [CrossRef]

76. Gutarowska, B.; Pietrzak, K.; Machnowski, W.; Milczarek, J.M. Historical Textiles-A Review of Microbial Deterioration Analysis and Disinfection Methods. Text. Res. J. 2017, 87, 2388-2406. [CrossRef]

77. Mazzoli, R.; Giuffrida, M.G.; Pessione, E. Back to the Past: "Find the Guilty Bug-Microorganisms Involved in the Biodeterioration of Archeological and Historical Artifacts". Appl. Microbiol. Biotechnol. 2018, 102, 6393-6407. [CrossRef]

78. Pangallo, D.; Kraková, L.; Chovanová, K.; Šimonovičová, A.; De Leo, F.; Urzì, C. Analysis and Comparison of the Microflora Isolated from Fresco Surface and from Surrounding Air Environment through Molecular and Biodegradative Assays. World J. Microbiol. Biotechnol. 2012, 28, 2015-2027. [CrossRef]

79. Kraková, L.; Šoltys, K.; Puškárová, A.; Bučková, M.; Jeszeová, L.; Kucharík, M.; Budiš, J.; Orovčík, L.; Szemes, T.; Pangallo, D. The Microbiomes of a XVIII Century Mummy from the Castle of Krásna Hôrka (Slovakia) and Its Surrounding Environment. Environ. Microbiol. 2018, 20, 3294-3308. [CrossRef] [PubMed]

80. Kisová, Z.; Planý, M.; Pavlović, J.; Bučková, M.; Puškárová, A.; Kraková, L.; Kapustová, M.; Pangallo, D.; Šoltys, K. Biodeteriogens Characterization and Molecular Analyses of Diverse Funeral Accessories from XVII Century. Appl. Sci. 2020, 10, 5451. [CrossRef]

81. Naji, K.M.; Abdullah, Q.Y.M.; AL-Zaqri, A.Q.M.; Alghalibi, S.M. Evaluating the Biodeterioration Enzymatic Activities of Fungal Contamination Isolated from Some Ancient Yemeni Mummies Preserved in the National Museum. Biochem. Res. Int. 2014, 2014, e481508. [CrossRef]

82. Kavkler, K.; Gunde-Cimerman, N.; Zalar, P.; Demšar, A. Fungal Contamination of Textile Objects Preserved in Slovene Museums and Religious Institutions. Int. Biodeterior. Biodegrad. 2015, 97, 51-59. [CrossRef]

83. Šimonovičová, A.; Kraková, L.; Pangallo, D.; Majorošová, M.; Piecková, E.; Bodoriková, S.; Dörnhoferová, M. Fungi on Mummified Human Remains and in the Indoor Air in the Kuffner Family Crypt in Sládkovičovo (Slovakia). Int. Biodeterior. Biodegrad. 2015, 99, 157-164. [CrossRef]

84. Šoltys, K.; Planý, M.; Biocca, P.; Vianello, V.; Bučková, M.; Puškárová, A.; Sclocchi, M.C.; Colaizzi, P.; Bicchieri, M.; Pangallo, D.; et al. Lead Soaps Formation and Biodiversity in a XVIII Century Wax Seal Coloured with Minium. Environ. Microbiol. 2020, 22, 1517-1534. [CrossRef] [PubMed]

85. Pangallo, D.; Kraková, L.; Chovanová, K.; Bučková, M.; Puškarová, A.; Šimonovičová, A. Disclosing a Crypt: Microbial Diversity and Degradation Activity of the Microflora Isolated from Funeral Clothes of Cardinal Peter Pázmány. Microbiol. Res. 2013, 168, 289-299. [CrossRef]

86. Hsu, S.C.; Lockwood, J.L. Powdered Chitin Agar as a Selective Medium for Enumeration of Actinomycetes in Water and Soil. Appl. Microbiol. 1975, 29, 422-426. [CrossRef]

87. Eggins, H.O.W.; Pugh, G.J.F. Isolation of Cellulose-Decomposing Fungi from the Soil. Nature 1962, 193, 94-95. [CrossRef]

88. Paterson, R.R.M.; Bridge, P.D.; International Mycological Institute. Biochemical Techniques for Filamentous Fungi; CAB International: Wallingford, UK, 1994.

89. Pangallo, D.; Bučková, M.; Kraková, L.; Puškárová, A.; Šaková, N.; Grivalský, T.; Chovanová, K.; Zemánková, M. Biodeterioration of Epoxy Resin: A Microbial Survey through Culture-Independent and Culture-Dependent Approaches. Environ. Microbiol. 2015, 17, 462-479. [CrossRef]

90. Shivani, D.; Kumar, J.S. Extracellular Enzymatic Profile of Fungal Deteriogens of Historical Palace of Ujjain. Int. J. Curr. Microbiol. Appl. Sci. 2015, 4, 122-132.

91. Strzelczyk, A.B. Observations on Aesthetic and Structural Changes Induced in Polish Historic Objects by Microorganisms. Int. Biodeterior. Biodegrad. 2004, 53, 151-156. [CrossRef]

92. Caneva, G.; Nugari, M.P.; Salvadori, O.; International Centre for the Study of the Preservation and the Restoration of Cultural Property. Biology in the Conservation of Works of Art; ICCROM-International Centre for the Study of the Preservation and Restoration of Cultural Property: Rome, Italy, 1991.

93. Puškárová, A.; Bučková, M.; Habalová, B.; Kraková, L.; Maková, A.; Pangallo, D. Microbial Communities Affecting Albumen Photography Heritage: A Methodological Survey. Sci. Rep. 2016, 6, 20810. [CrossRef]

94. Štafura, A.; Nagy, Š.; Bučková, M.; Puškárová, A.; Kraková, L.; Čulík, M.; Beronská, N.; Nagy, Š.; Pangallo, D. The Influence of Microfilamentous Fungi on Wooden Organ Pipes: One Year Investigation. Int. Biodeterior. Biodegrad. 2017, 121, 139-147. [CrossRef]

95. Abrusci, C.; Martín-González, A.; Del Amo, A.; Catalina, F.; Collado, J.; Platas, G. Isolation and Identification of Bacteria and Fungi from Cinematographic Films. Int. Biodeterior. Biodegrad. 2005, 56, 58-68. [CrossRef]

96. Rojas, T.I.; Aira, M.J.; Batista, A.; Cruz, I.L.; González, S. Fungal Biodeterioration in Historic Buildings of Havana (Cuba). Grana 2012, 51, 44-51. [CrossRef]

97. Kraková, L.; Chovanová, K.; Selim, S.A.; Šimonovičová, A.; Puškarová, A.; Maková, A.; Pangallo, D. A Multiphasic Approach for Investigation of the Microbial Diversity and Its Biodegradative Abilities in Historical Paper and Parchment Documents. Int. Biodeterior. Biodegrad. 2012, 70, 117-125. [CrossRef]

98. Anaya, M.; Borrego, S.F.; Gámez, E.; Castro, M.; Molina, A.; Valdés, O. Viable Fungi in the Air of Indoor Environments of the National Archive of the Republic of Cuba. Aerobiologia 2016, 32, 513-527. [CrossRef]

99. Borrego, S.; Molina, A.; Santana, A. Mold on Stored Photographs and Maps: A Case Study. Top. Photogr. Preserv. 2015, 16, 109-120. 
100. Borrego, S.; Molina, A.; Santana, A. Fungi in Archive Repositories Environments and the Deterioration of the Graphics Documents. EC Microbiol. 2017, 11, 205-226.

101. Saran, S.; Isar, J.; Saxena, R.K. A Modified Method for the Detection of Microbial Proteases on Agar Plates Using Tannic Acid. J. Biochem. Biophys. Methods 2007, 70, 697-699. [CrossRef] [PubMed]

102. Borrego, S.; Perdomo, I. Aerobiological Investigations inside Repositories of the National Archive of the Republic of Cuba. Aerobiologia 2012, 28, 303-316. [CrossRef]

103. Okpalanozie, O.E.; Adebusoye, S.A.; Troiano, F.; Polo, A.; Cappitelli, F.; Ilori, M.O. Evaluating the Microbiological Risk to a Contemporary Nigerian Painting: Molecular and Biodegradative Studies. Int. Biodeterior. Biodegrad. 2016, 114, 184-192. [CrossRef]

104. Boniek, D.; Bonadio, L.; Santos de Abreu, C.; Dos Santos, A.F.; de Resende Stoianoff, M.A. Fungal Bioprospecting and Antifungal Treatment on a Deteriorated Brazilian Contemporary Painting. Lett. Appl. Microbiol. 2018, 67, 337-342. [CrossRef]

105. Coronado-Ruiz, C.; Avendaño, R.; Escudero-Leyva, E.; Conejo-Barboza, G.; Chaverri, P.; Chavarría, M. Two New Cellulolytic Fungal Species Isolated from a 19 Th -Century Art Collection. Sci. Rep. 2018, 8, 7492. [CrossRef] [PubMed]

106. Teather, R.M.; Wood, P.J. Use of Congo Red-Polysaccharide Interactions in Enumeration and Characterization of Cellulolytic Bacteria from the Bovine Rumen. Appl. Environ. Microbiol. 1982, 43, 777-780. [CrossRef] [PubMed]

107. Pyzik, A.; Ciuchcinski, K.; Dziurzynski, M.; Dziewit, L. The Bad and the Good-Microorganisms in Cultural Heritage Environments-An Update on Biodeterioration and Biotreatment Approaches. Materials 2021, 14, 177. [CrossRef]

108. Piñar, G.; Sterflinger, K. Natural Sciences at the Service of Art and Cultural Heritage: An Interdisciplinary Area in Development and Important Challenges. Microb. Biotechnol. 2021. [CrossRef] [PubMed]

109. Leplat, J.; François, A.; Bousta, F. Parengyodontium album, a Frequently Reported Fungal Species in the Cultural Heritage Environment. Fungal Biol. Rev. 2020, 34, 126-135. [CrossRef]

110. O'Donnell, K.; Al-Hatmi, A.M.S.; Aoki, T.; Brankovics, B.; Cano-Lira, J.F.; Coleman, J.J.; de Hoog, G.S.; Pietro, A.D.; Frandsen, R.J.N.; Geiser, D.M.; et al. No to Neocosmospora: Phylogenomic and Practical Reasons for Continued Inclusion of the Fusarium Solani Species Complex in the Genus Fusarium. mSphere 2020, 5. [CrossRef] [PubMed]

111. Kraková, L.; Chovanová, K.; Puškarová, A.; Bučková, M.; Pangallo, D. A Novel PCR-Based Approach for the Detection and Classification of Potential Cellulolytic Fungal Strains Isolated from Museum Items and Surrounding Indoor Environment. Lett. Appl. Microbiol. 2012, 54, 433-440. [CrossRef] [PubMed]

112. Sterflinger, K.; Little, B.; Pinar, G.; Pinzari, F.; de los Rios, A.; Gu, J.-D. Future Directions and Challenges in Biodeterioration Research on Historic Materials and Cultural Properties. Int. Biodeterior. Biodegrad. 2018, 129, 10-12. [CrossRef] 